\title{
ODONATA COLLECTION OF THE CROATIAN NATURAL HISTORY MUSEUM
}

\author{
Marina Vilenica ${ }^{1, *}$ \& Iva Mihoci ${ }^{2}$ \\ ${ }^{1}$ Faculty of Teacher Education, University of Zagreb, Trg Matice hrvatske 12, 44250 Petrinja, Croatia \\ ${ }^{2}$ Croatian Natural History Museum, Demetrova 1, 10000 Zagreb, Croatia
}

Vilenica, M. \& Mihoci, I.: Odonata collection of the Croatian Natural History Museum. Nat. Croat., Vol. 27, No. 1, 153-184, Zagreb, 2018.

A total 1,545 Odonata specimens stored in the collection of the Croatian Natural History Museum in Zagreb have been examined. The specimens were collected in the period between 1869 and 2011, mainly in Croatia, but also in the surrounding countries (Austria, Bosnia and Herzegovina, Italy, Montenegro, Serbia, Slovenia) and Switzerland. An overview of 61 species is presented. The most numerous species were Ischnura elegans (Vander Linden, 1820) and Platycnemis pennipes (Pallas, 1771).

Keywords: collection, CNHM, Anisoptera, Zygoptera

Vilenica, M. \& Mihoci, I.: Zbirka vretenaca (Odonata) Hrvatskog prirodoslovnog muzeja. Nat. Croat., Vol. 27, No. 1, 153-184, Zagreb, 2018.

Pregledano je 1545 jedinki Odonata pohranjenih u zbirci Hrvatskog prirodoslovnog muzeja u Zagrebu. Najveći broj jedinki prikupljen je s područja Hrvatske, no nekolicina je prikupljena i na području susjednih država (Austrije, Bosne i Hercegovine, Crne Gore, Italije, Slovenije, Srbije) te Švicarske. Jedinke su prikupljene u periodu između 1869. i 2011. godine. Prikazana zbirka Odonata sadrži ukupno 61 vrstu. Najveći broj jedinki pripada vrstama Ischnura elegans (Vander Linden, 1820) i Platycnemis pennipes (Pallas, 1771).

Ključne riječi: zbirka, HPM, Anisoptera, Zygoptera

\section{INTRODUCTION}

Odonata are an ancient merolimnic insect order, originating approximately three hundred million years ago. Since then, they have changed slightly in structure and appearance (AsKew, 2004; Grimaldi \& ENGEL, 2005). Nowadays, this relatively small insect order is represented by approximately 6,300 species worldwide (SCHORR \& PAULson, 2018), about 143 of which inhabit European freshwater habitats (KALKMAN et al., 2008; Boudot \& Kalkman, 2015). Many studies have shown that Odonata are highly sensitive to small-scale environmental changes in their habitats, which is why they are widely used as model organisms in aquatic ecology research and conservation management (e.g. Moore, 1997; Suhling et al., 2006; Simaika \& Samways, 2008). Due to their attractive and distinctive appearance awakened human curiosity centuries ago (e.g. see in D'Aguilar et al., 1986; Belle, 1994).

The interest of researchers in the dragonfly fauna of Croatia and surrounding countries began in the first half of the $19^{\text {th }}$ century (CARRARA, 1846), and reached its peak

\footnotetext{
*Corresponding author: marina.vilenica@gmail.com; marina.vilenica@ufzg.hr
} 
during the last quarter of the $20^{\text {th }}$ century (see in BELANčić et al., 2008) and the beginning of the $21^{\text {st }}$ century (e.g. Perović \& Perović, 2006; Bogdanović et al., 2008; Vilenica et al., 2011; Vilenica \& DijKstRa, 2014; ŠTih et al., 2015; Vilenica et al., 2016). However, many highly valuable data stored in personal collections or those of natural history museums have not been published. Moreover, collections in natural history museums often contain specimens collected several decades ago (e.g. Jović \& MinajLova, 2009; ŠAšić et al., 2016). Comparison of historical data and the fauna present today at a specific habitat could contribute to the detection of habitat alterations over a long-term period, as well as to species protection and management of important areas. Therefore, collections are of essential importance in faunistic studies and biodiversity conservation.

The Croatian Natural History Museum, whose predecessor the National Museum was founded in 1846 in Zagreb, is one of the oldest and largest of Croatian museums. Since then, it has had an important role in collecting, guarding and studying various natural treasures (e.g. minerals, rocks, fossils, extant plants, animals and anthropological remains) from Croatia and neighbouring countries. Nowadays, about 1.5 million specimens systematized in 117 collections are stored there. In the beginnings, Odonata specimens stored in the CNHM were not a part of a particular insect collection. Many of them were collected during research activities of numerous biologists, museum entomologists and other museum collaborators (V. Bartovski, Z. Bokulić, D. Ćaleta, V. Dumbović, M. Franković, G. Gjerapić, Halapir, D. Holcer, B. Jalžić, V. Jelenković, E. Kletečki, Gj. Koča, M. Kučinić, S. Leiner, G. Lukač, A. Magerle, R. Ozimec, I. Pavlinić, F. Perović, G. Perović, N. Polašek, D. Radović, Z. Ružanović, V. Ščetarić, T. Šoljan, M. Stipčević, V. Strpić, N. Tvrtković, M. Vajdić, I. von Igalffy, S. Vujčić-Karlo, A. Vukušić, S.V. Weingartner). Finally, all specimens where systematically ordered to create a separate and unique Odonata collection of the CNHM. Nowadays, the Odonata collection, founded by museum advisor (curator) Franjo Perović, has 1.625 specimens. A part of the collection, related to Odonata of Međimurje County, Croatia, has already been reported by Perović \& Perović, 2006. Most specimens originating from Bosnia and Herzegovina were included in the review of the Odonata of Bosnia and Herzegovina given by Kulijer et al. (2013). Our main goal was to give a complete list of Odonata species and specimens kept in the Croatian Natural History Museum.

\section{MATERIALS AND METHODS}

We examined a total of 1,625 Odonata specimens collected between 1869 and 2011, mainly from Croatia, but including some specimens collected from Austria, Bosnia and Herzegovina, Italy, Montenegro, Serbia, Slovenia and Switzerland. The majority of specimens dating back to the last three decades of the $20^{\text {th }}$ century and the first decade of the $21^{\text {st }}$ century were collected by museum advisor (curator) Franjo Perović. On the other hand, the collectors of the majority of specimens accessioned between the late $19^{\text {th }}$ and early $20^{\text {th }}$ century are not known.

Identifications of historical records were rather challenging, because of the involved history of toponyms in this part of Europe. Therefore, we had to consult various resources (e.g. the historical maps of the region). The most demanding question was the location of the locality Mitrovica. There was a possibility the specimens with "Mitrovica" labels were collected in Kosovska Mitrovica in Kosovo, or Sremska Mitrovica in Serbia. Nevertheless, analysis of other localities visited in April 1911 revealed a number of Odonata specimens collected around the same time at nearby localities in Serbia 
(such as Bačinci village). Moreover, in the topographic map created in the $3^{\text {rd }}$ Military Mapping Survey of Austria-Hungary, Sremska Mitrovica town was designated as "Mitrowitz (Mitrovica)". Therefore, we concluded there is a higher possibility that this specimen was collected in Serbia and not in Kosovo.

A number of specimens had labels with the identification of the species. These identifications were made by the Croatian odonatologists, such as Dr. Matija Franković and Nino Mihoković, former museum staff, such as museum advisor (curator) Franjo Perović and biology students. Specimens with such labels were re-examined; their identifications were confirmed or in some cases corrected, while the previously unidentified material was identified using Askew (2004) and DijKstra \& Lewington (2006).

The nomenclature we used in creating this overview follows DiJKSTRA \& LEWINGTON (2006). We present data for 1,545 specimens for which we were able to determine the localities where they were collected. As for the remaining 80 specimens stored in the collection, the names of the localities could either not be deciphered reliably or else were not marked on the labels, and hence they were excluded from the review. For each species, localities are presented in alphabetical order.

\section{RESULTS AND DISCUSSION}

The presented Odonata collection comprises 61 species (with 1,545 specimens), which represents $43 \%$ of the European Odonata fauna (KALKMAN et al., 2008; Boudot \& KaLKMAN, 2015). The most represented families were the largest (i.e. most speciose) European families (Dijkstra \& Lewington, 2006), Libellulidae (with 18 species) and Coenagrionidae (with 12 species). Eurytopic Ischnura elegans (Vander Linden, 1820) and Platycnemis pennipes (Pallas, 1771), species with wide ecological tolerance (DijKstra \& Lewington, 2006) were present with the highest number of individuals (Fig. 1). The collection contains one male of Sympetrum danae (Sulzer, 1776), collected at an unknown locality, and one male of Lestes macrostigma (Eversmann, 1836) collected at a locality we were not able to pinpoint. Therefore, we have not managed to determine where the specimens/species originate from. Both species are considered as doubtful for Croatian fauna (BELANčić et al., 2008, Kotarac et al., 2016) and might have been collected in one or other of the surrounding countries (see in e.g. Kotarac, 1997; Kulijer et al., 2013).

As expected, the highest number of species and specimens were collected in Croatia, (i.e. a total of 58 species, all except Somatochlora metallica (Vander Linden, 1825), and most likely Sympetrum danae and Lestes macrostigma), which represents $84 \%$ of Croatian Odonata fauna (Belančić et al., 2008; KotARAc et al., 2016). The last Odonata inventory in Croatia (KotARAc et al., 2016) recorded four additional species that are missing from the Museum's collection: Caliaeschna microstigma (Schneider, 1845), Gomphus flavipes (Charpentier, 1825), Leucorrhinia caudalis (Charpentier, 1840) and Cordulegster heros Theischinger, 1979. On the other hand, the collection contains some species that were not recorded in the recent inventories (VINKO \& Vilenica, 2013; KotaRAc et al., 2016), such as Sympetrum pedemontanum (Müller in Allioni, 1766) (Fig. 2). As the specimens in the collection originate from the state border between Croatia and Slovenia, it is possible that the species' place of emergence was in Slovenian habitats (KотARAc et al., 1997; VINKO, 2017) and that it arrived in the neighbouring Croatia in search for food.

Furthermore, ten species were recorded from both Bosnia and Herzegovina (Lestes sponsa (Hansemann, 1823), Lestes virens (Charpentier, 1825), Lestes parvidens Artobolevskii, 1929, Ischnura elegans, Aeshna affinis Vander Linden, 1820, Aeshna cyanea (Müller, 


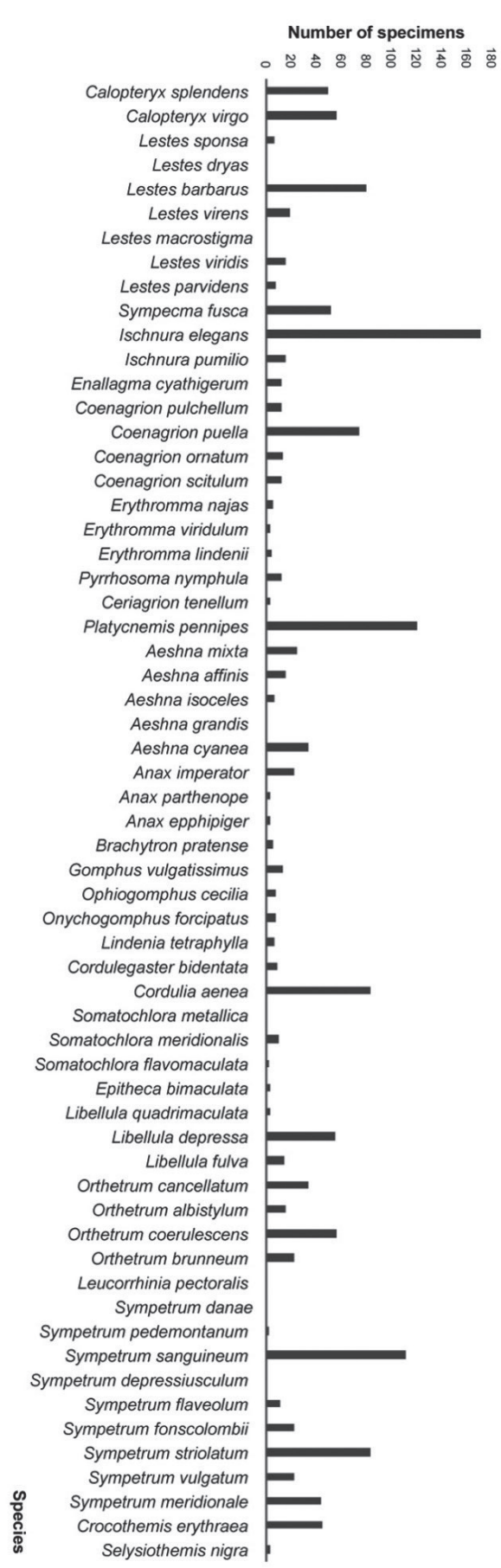

Fig. 1. Dragonfly species overview with number of specimens in the Odonata collection of the Croatian Natural History Museum, Zagreb.

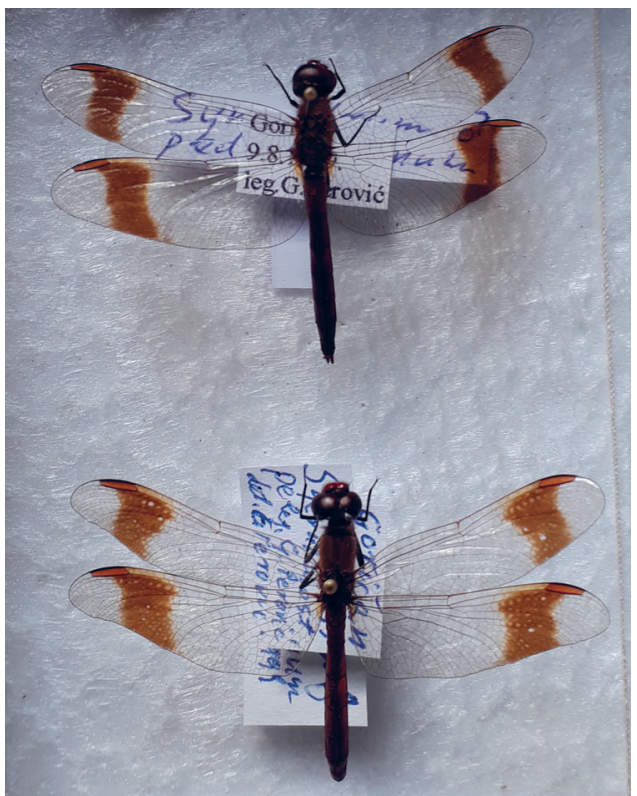

Fig. 2. Sympetrum pedemontanum (Müller in Allioni, 1766) specimens from the Odonata collection of the Croatian Natural History Museum, Zagreb.

1764), Anax imperator Leach, 1815; Sympetrum sanguineum (Müller, 1764), Sympetrum flaveolum (Linnaeus, 1758), Sympetrum striolatum (Charpentier, 1840)) and Serbia (Lestes sponsa, Lestes barbarus (Fabricius, 1798), Sympecma fusca (Vander Linden, 1820), Ischnura elegans, Ischnura pumilio (Charpentier, 1825), Coenagrion scitulum (Rambur, 1842), Orthetrum albistylum (Sélys, 1848), Orthetrum brunneum (Fonscolombe, 1837), Sympetrum sanguineum, Sympetrum meridionale (Sélys, 1841)), five species from Montenegro (Sympecma fusca, Aeshna affinis, Somatochlora metallica, S. meridionale, Calopteryx splendens (Harris, 1780)), two species from Slovenia (A. cyanea, Cordulegaster bidentata Sélys,1843), while only one species was collected in Austria (Orthetrum cancellatum (Linnaeus, 1758)), Italy (Crocothemis erythraea (Brullé, 1832)) and Switzerland (Sympetrum flaveolum (Linnaeus, 1758)).

A number of species stored in the collection are of international conservation concern (KalKman et al., 2010). Lindenia tetraphylla (Vander Linden, 1825) is listed in Annexes II 
and IV of the Habitats Directive and was considered vulnerable in the European Red List. Ophiogomphus cecilia (Fourcroy, 1785) and Leucorrhinia pectoralis (Charpentier, 1825) are listed in Annexes II and IV of the Habitats Directive, and Coenagrion ornatum (Sélys, 1850) in Annex II. Lestes macrostigma is a vulnerable taxon in the European Red List. Moreover, C. ornatum, L. pectoralis, O. cecilia and L. tetraphylla are Natura 2000 species (Kotarac et al., 2016). Additionally, a number of species are protected or strictly protected taxa in Croatia (Narodne novine, No. 80/13, 15/18), as well as listed in the Croatian Red List (BELANČić et al., 2008).

\section{Species list:}

\section{Suborder: ZYGOPTERA Sélys, 1854}

\section{Family: CALOPTERYGIDAE}

\section{Calopteryx splendens (Harris, 1780)}

The species has wide European distribution, inhabiting partially open lotic habitats (AsKew, 2004; Dijkstra \& Lewington, 2006). In the collection, it is represented by a total of 49 individuals, 48 of which were collected at 24 localities in Croatia (CRO) and one individual at one locality in Montenegro (MNE).

CRO: Belec village (1 female, 05.08.2009, leg. unknown); Blace village, Neretva River mouth (1 male, 29.04.1996, leg. F. Perović); Domašinec municipality, Murščak forest, canal (1 female, 03.08.1999, leg. F. Perović); Draganić municipality (1 male, 13.06.1964, leg. unknown); Goričan village, Črni most bridge, canal (1 male, 15.05.2004, leg. F. Perović); Krka National Park (1 male, 1 female, 15.08.1999, leg. B. Jalžić); Krka River (1 female, 25.06.1996, leg. F. Perović); Kumrovec village (1 male, 24.06.1996, leg. F. Perović); Međimurje County, Šenkovec village (1 male, 06.09.2004, leg. F. Perović); Mura River (1 male, 23.06.1999, leg. F. Perović); Murščak forest, canal (3 males, 1 female, 16.05.1999, 22.06.1999, 26.06.2005, leg. F. Perović); Murščak forest, Mura River (1 female, 17.05.1999, leg. G. Gjerapić); Neretva River, Modro oko Lake (2 males, 2 females, 05.06.1993, 01.08.1997, leg. F. Perović; 1 male, 01.08.1997, leg. G. Gjerapić); Novakovec village (1 male, 22.06.1999, leg. F. Perović); Pešćenica village, Odra River (1 male, 1 female, 08.03.1977, leg. unknown); Petrinja town (1 male, 2 females, 25.05.1907, leg. unknown); Temovec village (1 male, 1 female, 07.1942, leg. I. von Igalffy); Turopolje wetland, Vratovo locality (1 male, 1 female, 04.05.1997, leg. F. Perović; 1 female, 27.05.1998, leg. M. Kučinić); Zagreb city (1 male, 1 female, 25.06.1908, leg. unknown); Zagreb city, Jarun Lake (1 female, 04.06.1992, leg. Z. Bokulić); Zagreb city, Maksimir (2 males, 22.06.1952, leg. unknown); Zagreb city, Mirogoj (1 female, 06.06.1905, leg. unknown); Zagreb city, Sava River (2 males, 3 females, 11.06.1992, leg. unknown); Zapeć village (5 females, 07.1909, leg. unknown).

MNE: Kotor town (1 male, 07.1931, leg. unknown).

\section{Calopteryx virgo (Linnaeus, 1758)}

The species has wide European distribution, preferably inhabiting cooler, smaller and shaded lotic habitats, most typically small forest streams (AsKEw, 2004; DiJKSTRA \& LewINGTON, 2006). In the collections, it was represented by a total of 56 individuals, collected at 30 localities in Croatia. 
CRO: Brod na Kupi - Kuželj villages (1 male, 1 female, 09.06.2004, leg. F. Perović); Cetina River, Balečki bridge (1 male, 1 female, 27.07.1985, leg. M. Franković); Draškovec village (1 male, 1 female, 28.05.1999, leg. F. Perović); Drava River oxbow Halaševo (24 km) (1 female, 19.07.1985, leg. M. Franković); Dretulja river spring (Vrelske bare) (1 female, 30.07.2004, leg. N. Tvrtković); Gorski Kotar region, stream (2 males, 2 females, 19.06.1998, leg. M. Kučinić); Jablanac village (1 male, 10.08.1908, leg. unknown); Jagnjedovac village (1 male, 18.07.1900, leg. unknown); Jelinac village (1 male, 12.08.1982, leg. unknown); Krbavsko polje, Krbavica River (1 female, 02.07.2004, leg. I. Pavlinić); Krka National Park, Roški slap waterfall (4 males, 1 female, 21.05.1985, leg. unknown); Lika region, Stajničko polje, spring (1 male, 28.06.2004, leg. F. Perović); Lipovac village (1 male, 02.08.1918, leg. unknown); Lonjsko polje wetland, canal (1 male, 05.08.1982, leg. unknown); Mljet Island, Malo jezero Lake (2 females, 12.08.1982, leg. unknown); Motovun village, Butoniga Reservoir (1 male, 16.06.1988, leg. D. Radović); Murščak forest, canal (1 female, 26.06.2005, leg. F. Perović); Neretva River, Modro oko Lake (1 female, 01.08.1977, leg. F. Perović; 1 female, 09.08.1977, leg. M. Kučinić); Ogulin town (1 male, 29.06.1910.; 1 male, 04.06.1998, legs. unknown); Pešćenica village, Koravec stream (1 male, 20.05.2002, leg. F. Perović); Risnjak Mountain (1 male, 14.07.1903, leg. unknown); Sedalce village, Kupa River upper reaches (3 females, 22.07.2004, leg. F. Perović); Temovec village (2 males, 1 female, 07.1942, leg. I. von Igalffy); Valentinovo village, Bežanec castle (1 female, 07.1992, leg. unknown); Zagreb city (1 male, 07.1869, leg. unknown); Zagreb city, Čučerje (1 male, 21.05.1997, leg. unknown); Zagreb city, Maksimir (1 male, date and leg. unknown; 1 female, 22.06.1952, leg. unknown); Gorski Kotar region, Zapeć village, Kupa River (5 males, 3 females, 07.1909, leg. unknown); Žumberak Mountain, Slapnica River (2 males, date unknown, leg. M. Kučinić; 25.06.1992, leg. M. Kučinić); Zvečevo village (1 male, 24.07.1996, leg. unknown).

\section{Family: LESTIDAE}

\section{Lestes sponsa (Hansemann, 1823)}

The species is one of the most common species in a large part of Northern Europe, while it is mainly absent from most of the southern area. It inhabits the majority of lentic habitats with reed-like vegetation (Askew, 2004; Dijkstra \& Lewington, 2006). In the collections, it was represented by a total of seven individuals, four of which were collected at two localities in Croatia (CRO), two at one locality in Bosnia and Herzegovina (B\&H) and one individual at one locality in Serbia (SER). The species is a protected (NARODNE NOvine, No. 80/13, 15/18) and nearly threatened taxon in Croatia (BELANČıć et al., 2008).

CRO: Đurđevac village (1 male, 21.07.1901, leg. unknown); Selce village (1 male, 2 females, 10.07.1986, leg. B. Jalžić).

B\&H: Livanjsko polje, Kazanci-Provo (1 male, 1 female, 25.07.1987, leg. F. Perović).

SER: Kupinovo village (1 female, 19.07.1902, leg. unknown).

\section{Lestes dryas Kirby, 1890}

The species has Holarctic distribution, being common in Europe and North America. In Europe, it is more common in the northern and central part, but less frequent than $L$. sponsa. Additionally, it is more common southwards than the former species. It inhabits a wide range of lentic intermittent habitats (AsKEw, 2004; DiJKsTRA \& Lewington, 2006). In the collections, it was represented with only one individual, collected from a pond at 
the state border between Croatia and Slovenia: Goričan border crossing (Croatia-Slovenia), pond (1 female, 25.06.2005, leg. F. Perović). The species is a protected (NArodne NOvine, No. 80/13, 15/18) and nearly threatened taxon in Croatia (BELANčić et al., 2008).

\section{Lestes barbarus (Fabricius, 1798)}

In Europe, the species is most common in the Mediterranean part, less common in the central part, while it is very rare in its northern part. It preferably inhabits intermittent lentic habitats that dry out in early summer (AsKew, 2004; DiJKSTRA \& LEWINGTON, 2006). In the collection, it is represented by a total of 80 individuals, 76 of which were collected at 18 localities in Croatia (CRO) and four individuals at two localities in Serbia (SER). The species is a protected (NARodne novine, No. 80/13, 15/18) and nearly threatened taxon in Croatia (Belančić et al., 2008).

CRO: Blace village, Neretva River mouth (1 female, 10.09.1997, leg. V. Strpić); Dijaneš village (1 male, date and leg. unknown); Draganić municipality (2 females, 03.06.1964, leg. A. Magerle); Hvar Island, Starigrad village (2 females, 08.1910, leg. unknown); Lukavec village (1 female, 16.06.07, leg. unknown); Osijek city (1 male, 2 females, 03.07.1901, 05.07.1902, 15.07.1997, legs. unknown); Otočac town (1 male, 18.07.1912, leg. unknown); Pag Island, Potočnica village, ponds (1 male, 07.07.2001, leg. F. Perović); Pag Island, Caska village (1 female, 23.06.1956, leg. unknown; 1 female, 06.10.1955, leg. A. Magerle; 2 females, 27.06.1960, leg. unknown; 7 females, 13 males, 09.06.1958, leg. unknown; 2 females, 08.06.1958, leg. unknown; 5 females, 12 males, 12.06.1958, leg. unknown; 1 male, 06.10.1955, leg. unknown; 2 males, 1 female, 31.05.2002, leg. F. Perović; 1 female, 31.08.2001, leg. F. Perović); Pag Island, Kolansko blato wetland (2 males, 3 females, 11.06.1958, leg. unknown); Pag Island, Novalja town, Vidasi (1 female, 28.07.1996, leg. F. Perović); Paklenica National Park, canyon of the Stražbenica River (1 female, 24.06.1985, leg. F. Perović); Petrinja town (1 female, 24.05.1907, leg. unknown); Poreč town (1 male, 11.08.1956, leg. unknown); Vinkovci town (1 male, 30.08.1912, leg. unknown); Vis Island, Podšpilje village, pond (4 females, 13.06.1996, leg. F. Perović); Zadar County, Ljubač village, on the blackberry bushes (1 male, 28.09.1985, leg. G. Lukač); Zagreb city (1 male, date and leg. unknown).

SER: Ogar village (1 male, 1 female, 11.08.2013, leg. unknown; 1 male, 12.06.1958, leg. unknown); Subotište village (1 female, 25.07.1902, leg. unknown).

\section{Lestes virens (Charpentier, 1825)}

The species has a mainly southern and central European distribution, while it is also present in North Africa. It inhabits a wide range of seasonally dry lentic habitats in the south and bogs in the north (AsKew, 2004; Dijkstra \& Lewington, 2006). In the collection, it is represented by a total of 19 individuals, ten of which were collected at six localities in Croatia (CRO) and nine at four localities in Bosnia and Herzegovina (B\&H). The species is a strictly protected (NARodne novine, No. 80/13, 15/18) and vulnerable taxon in Croatia (Belančić et al., 2008).

CRO: Osijek city (1 female, 05.07.1902, leg. unknown); Pag Island, Caska village (1 male, 1 female, 03.05.2001, leg. F. Perović; 1 male, 1 female, 06.07.2001, leg. F. Perović; 1 female, 07.07.2001, leg. F. Perović); Pag Island, Novalja town (1 male, 28.08.2001, leg. F. Perović), Sunja village (1 male, 30.08.2011, leg. F. Perović); Turopolje wetland, Greda village (1 male, 09.07.1986, leg. F. Perović); Velebit Mountain, Štirovac summit (1 female, 25.07.1917, leg. unknown). 
B\&H: Dinara Mountain, Goleš summit, 1550 m (1 male, 1 female, 26.05.1985, leg. M. Franković; 2 males, 26.07.1985, leg. M. Franković); Dinara Mountain, Poljarice-Troglav (1 male, 24.07.1987, leg. S. Leiner); Dinara Mountain, Poparić pond, $1250 \mathrm{~m}$ (1 male, 1 female, 23.07.1987, leg. S. Leiner and F. Perović); Livanjsko polje, Kazanci-Provo (2 females, 25.07.1987, leg. F. Perović).

\section{Lestes macrostigma (Eversmann, 1836)}

The species has a disjunct distribution in Europe, being absent from most of Northern and Central Europe, and more common towards the south. It could be solely found in shallow lentic habitats with the dense rushes of coastal and saline inland wetlands (DijKstra \& LEWINGTON, 2006). In the collection, the species was represented with one male collected on July $17^{\text {th }} 1902$ at the locality named "Osijevac", which we were not able to locate.

\section{Lestes viridis (Vander Linden, 1825)}

The species is widespread in Southern and Central Europe, also occurring in Africa, Georgia, and the Middle East. It inhabits a wide range of lentic and slow flowing lotic habitats with surrounding trees and bushes, avoiding intermittent conditions (AsKEw, 2004; DijKstra \& Lewington, 2006). In the collection, it is represented by a total of 15 individuals collected at eight localities in Croatia (CRO).

CRO: Konjšćina village (1 male, 09.07.1993, leg. unknown); Osijek city (1 male, 03.08.1998, leg. unknown); Štrigova village (1 female, 07.08.1954, leg. unknown); Temovec village (1 female, 09.1924, leg. I. von Igalffy); Zagreb city (1 male, 1 female, date and leg. unknown), Zagreb city, Maksimir (2 females, 21.09.1954, leg. unknown); Zagreb city, Prevlaka village, Odra river (3 females, 2 males, 23.09.1985, leg. M. Franković); Zagreb city, Sava River (2 males, 01.10.05, leg. unknown).

\section{Lestes parvidens Artobolevskii, 1929}

The species' distribution is still not sufficiently known, but the most abundant populations were recorded in the Balkan Peninsula and Italy. It inhabits a wide range of lentic and slow flowing lotic habitats with surrounding trees and bushes (DIJKSTRA \& LEWINGTON, 2006). In the collection, it is represented by a total of eight individuals, seven of which were collected at four localities in Croatia (CRO) and one individual at one locality in Bosnia and Herzegovina $(\mathrm{B} \& \mathrm{H})$. The species is strictly protected (NAROdNE Novine, No. 80/13, 15/18) and data deficient taxon in Croatia (Belančić et al., 2008).

CRO: Domašinec municipality, Murščak forest, canal (1 male, 2 females, 15.10.2005, leg. F. Perović); Domašinec municipality, Murščak forest, pond (1 male, 1 female, 03.08.1998, leg. G. Gjerapić); Prekodravlje area, Repaš village, Čambina hunting lodge (1 male, 05.10.2001, leg. F. Perović); Sesvete village, stream (1 male, 04.07.2004, leg. M. Perović).

B\&H: Miljacka River (1 male, 27.10.2001, leg. F. Perović).

\section{Sympecma fusca (Vander Linden, 1820)}

The species is common in most of Europe but scarce in its northern part. Its distribution extends to Central Asia and North Africa. It inhabits a wide range of lentic habitats with well-developed aquatic vegetation (AsKew, 2004; DiJKsTRA \& LEWINGTON, 
2006). In the collection, it is represented by a total of 52 individuals, 45 of which were collected at 22 localities in Croatia (CRO), one at one locality in Montenegro (MNE), and six individuals at four localities in Serbia (SER).

CRO: Božjakovina village (1 female, 06.05.1997, leg. unknown); Cerje village, pond (1 female, 07.09.2003, leg. F. Perović); Fužine village (1 female, 28.08.1998, leg. F. Perović); Jablanovec village (1 female, 10.1908, leg. unknown); Koprivnica town (1 female, 19.07.1901, leg. unknown); Krka National Park, Visovac Lake (2 females, 25.04.1989, leg. D. Radović); Krndija Mountain (1 male, 2 females, 27.07.1916, leg. unknown); Metković town, Neretva River (1 female, 01.05.1996, leg. unknown); Motovun village, Butoniga Reservoir (4 males, 1 female, 16.06.1988, leg. D. Radović); Murščak forest, pond (1 female, 17.05.1999, leg. F. Perović); Osijek city (1 male, 13.04.1997, leg. unknown; 2 males, 1 female, 29.03.1998, leg. unknown; 1 male, 07.04.1998, leg. unknown); Pag Island, Caska village (1 male, 1 female, 19.04.2003, leg. F. Perović; 1 female, 02.05.2003, leg. F. Perović); Pag Island, Stara Novalja village (1 female, 04.05.2001, leg. F. Perović); Paklenica Mountain (1 female, 18.07.1911, leg. unknown; 1 female, 20.06.1999, leg. G. Gjerapić); Papuk Mountain, Jovanovica stream (1 male, 17.09.1986, leg. F. Perović; 1 female, 16.10.1986, leg. B. Jalžić); Plješevica Mountain (1 male, 15.08.1918, leg. unknown); Šenkovec village, pond (1 male, 01.08.2009, leg. F. Perović); Southern Velebit Mountain, Močila village, Borisov dom, 650 m a.s.l. (1 female, 17.07.1985, leg. F. Perović); Temovec village (2 males, 09.1924, leg. I. von Igalffy); Turopolje wetland, Turopoljski lug, Prevlaka (1 male, 12.09.1986, leg. F. Perović); Zagreb city (1 male, 1 female, date and leg. unknown; 1 male, 25.08.1899, leg. unknown; 2 females, 13.09.1899, leg. unknown; 1 female, 21.07.1905, leg. unknown); Zagreb city, Savica (3 males, 1 female, 04.05.1986, leg. D. Radović).

MNE: Cetinje city (1 female, 08.08.2003, leg. F. Perović).

SER: Bačinci village (1 female, 29.04.1911, leg. unknown); Kupinovo village (1 female, 19.07.1902, leg. unknown); Sremska Mitrovica town (1 female, 25.04.1911, leg. unknown); Zemun town (Belgrade city) (3 females, 19.05.1912, leg. unknown).

\section{Family: COENAGRIONIDAE}

\section{Ischnura elegans (Vander Linden, 1820)}

The species has wide distribution, extending from Ireland to Japan. It is one of the most ubiquitous species, inhabiting a wide range of lentic and slow flowing lotic habitats (AsKew, 2004; DijKstra \& Lewington, 2006). In the collection, it is represented by a total of 171 individuals, 166 of which were collected at 51 localities in Croatia (CRO), two at one locality in Bosnia and Herzegovina $(\mathrm{B} \& \mathrm{H})$ and three individuals at two localities in Serbia (SER).

CRO: Blace village (1 female, 29.04.1996, leg. unknown; 1 female, 29.07.1997, leg. V. Ščetarić); Blace village, reeds (1 male, 1 female, 28.07.1997, leg. F. Perović), Borovci municipality, Neretva River (1 female, 08.08.1997, leg. M. Kučinić); Draganić municipality (1 female, 13.05.1975, leg. F. Perović; 2 males, 19.05.1975, leg. F. Perović); Glušci village, Neretva River (2 males, 10.09.1997, leg. Vajdić and V. Strpić); Goričan village, canal by the Mura River (1 male, 23.05.1999, leg. F. Perović); Goričan village, Trnava River, Črni bridge (1 male, 2 females, 25.08.2000, leg. F. Perović); Hvar Island, Starigrad village (2 males, 08.1910, leg. unknown); Jurovčak village (1 male, 1 female, 20.05.2001, leg. F. Perović); Kosa-Dubravice villages, Neretva River (3 males, 03.08.1996, leg. G. 
Gjerapić); Križovec village (3 males, 2 females, 12.08.1999, leg. F. Perović; 1 male, 25.04.2000, leg. F. Perović); Križovec village, fishpond (2 males, 5 females, 23.05.1999, leg. F. Perović; 1 female, 22.06.1999, leg. F. Perović, 1 female, 17.03.2002, leg. F. Perović; 3 males, 1 female, 29.05.2004, leg. F. Perović); Krk Island (1 male, 17.06.1998, leg. J. Rota); Krk Island, Čižići village (2 males, 03.05.1992, leg. F. Perović); Krk Island, Njivice Reservoir (1 male, 1 female, 18.08.1926, leg. unknown); Krka National Park, Čulišićke bare ponds (1 female, 03.07.1989, leg. M. Stipčević); Krka National Park, Visovac Lake (8 males, 4 females, 25.04.1989, leg. D. Radović); Lonjsko polje wetland, canal by the overpass (1 male, 02.08.1982, leg. unknown); Lonjsko polje wetland, bank by the canal (1 male, 02.08.1982, leg. unknown); Lonjsko polje wetland, canal by the road (1 male, 02.08.1982, leg. unknown); Lonjsko polje wetland, flooded meadow (1 male, 1 female, 03.08.1982, leg. unknown); Mala Neretva River (1 male, 30.07.1997, leg. V. Ščetarić); Mirna River (1 male, 1 female, 22.06.1986, leg. F. Perović); Mislina village, Neretva River (1 male, 1 female, 03.08.1996, leg. G. Gjerapić); Modro oko Lake (1 female, 04.08.1996, leg. M. Kučinić; 1 male, 1 female, 06.08.1996, leg. M. Kučinić; 2 males, 01.08.1997, leg. G. Gjerapić; 1 male, 01.08.1997, leg. V. Ščetarić; 3 females, 09.08.1997, leg. M. Kučinić; 1 male, 08.04.1999, leg. F. Perović); Neretva River mouth (1 female, 09.1948, leg. unknown; 1 male, 01.08.1997, leg.V. Ščetarić); Neretva River, Desne Lake, (4 males, 3 females, 07.04.1999, leg. F. Perović); Neretva River, Kuti Lake (1 female, 03.07.1996, leg. M. Kučinić); Neretva River, Modro oko Lake, quarry (1 female, 11.08.1997, leg. M. Kučinić); Osijek city (1 male, 13.07.1901, leg. unknown; 1 male, 2 females, 22.08.1998, leg. unknown); Pag Island (5 males, 07.06.1907, leg. unknown); Pag Island, Caska village (1 female, 11.04.1960, leg. unknown; 1 male, 1 female, 19.04.2003, leg. F. Perović); Pag Island, Kolansko blato wetland (4 males, 1 female, 11.06.1958, leg. unknown); Pag Island, Novalja town, Vidasi (1 male, 4 females, 28.07.1996, leg. G. Gjerapić; 6 males, 5 females, 03.05.2001, leg. F. Perović; 1 male, 17.04.2003, leg. F. Perović); Pag Island, Potočnica village, ponds (2 males, 2 females, 07.07.2001, leg. F. Perović); Pag Island, Povljana village (1 male, 10.1960, leg. unknown); Pag Island, Stara Novalja village (1 male, 04.05.2001, leg. F. Perović); Pag Island, Velo blato Lake (1 male, 3 females, 05.05.2001, leg. F. Perović; 5 males, 1 female, 18.04.2003, leg. F. Perović); Pešćenica village (1 female, 08.05.1977, leg. unknown); Repušnica village (2 females, 21.05.1983, leg. unknown); Seline village, Neretva River (1 male, 11.08.1997, leg. M. Kučinić); Selnica village, fishpond (1 male, 23.05.1999, leg. F. Perović; 1 male, 1 female, 12.08.1999, leg. F. Perović); Šenkovec village (1 male, 30.08.2009, leg. F. Perović); Velika Gorica town (1 male, 16.04.1981, leg. unknown); Vinkovci town (2 males, 31.08.1912, leg. unknown); Vransko jezero Lake (2 males, 04.06.1986, leg. unknown); Vrpolje village (1 male, 15.07.1912, leg. unknown); Zagreb city (1 male, date and leg. unknown); Zagreb city, Bundek Lake (1 male, 24.05.1985, leg. unknown); Zagreb city, Sava River (1 male, 12.07.1992, leg. Z. Bokulić; 1 male, 23.08.1992, leg. Z. Bokulić; 1 male, 25.08.1992, leg. Z. Bokulić); Zagreb city, Savica (1 male, 30.04.1986, leg. D. Radović; 6 males, 1 female, 02.05.1986, leg. D. Radović); Zagreb city, Prevlaka village, Odra River (1 male, 23.09.1985, leg. M. Franković).

B\&H: Boljevići village (2 males, 20.05.1912, leg. unknown).

SER: Ogar village (1 male, 1 female, 11.08.1913, leg. unknown); Stara Pazova town (1 male, 22.07.1902, leg. unknown). 


\section{Ischnura pumilio (Charpentier, 1825)}

The species can be found over a large part of Europe in scattered colonies but is most numerous in the south. It has also ben recorded from western North Africa. It preferably inhabits recently created habitats with sparse vegetation (e.g. quarries) but it can also be found in various ponds, springs and ditches (AsKew, 2004; DijKsTRA \& LewiNGTON, 2006). In the collection, it is represented by a total of 16 individuals, 15 of which were collected at ten localities in Croatia (CRO) and one individual at one locality in Serbia (SER).

CRO: Cetina River, Balečki bridge (1 male, 27.07.1985, leg. M. Franković); Jurovčak village (1 male, 1 female, 15.05.2005, leg. F. Perović); Lonjsko polje wetland (1 male, 02.08.1982, leg. unknown; 1 male, 28.04.1983, leg. unknown); Lonjsko polje wetland, bank by the canal (1 male, 02.08.1982, leg. unknown); Northern Velebit National Park, Krasnarska jezera grasslands, 1500 m a.s.l. (1 male, 28.06.1983, leg. F. Perović); Pag Island, Caska village (2 males, 19.04.2003, leg. F. Perović); Pag Island, Novalja town, Vidasi (1 female, 28.07.1996, leg. G. Gjerapić; 2 females, 03.05.2001, leg. G. Perović); Šenkovec village, Lateralac stream (1 female, 19.06.1999, leg. F. Perović); Vugrovec village (1 male, 08.1942, leg. unknown); Zagreb city (1 male, date and leg. unknown).

SER: Sremska Mitrovica town (1 female, 27.04.1911, leg. unknown).

\section{Enallagma cyathigerum (Charpentier, 1840)}

The species is widely distributed in Europe, North America and in Asia as far east as Mongolia. It inhabits a wide range of lentic habitats (ponds, lakes) and slow rivers (Askew, 2004; Dijkstra \& Lewington, 2006). In the collections, it was represented by a total of 12 individuals collected at eight localities in Croatia (CRO).

CRO: Belec village (1 male, 1 female, 05.08.1909. leg. unknown); Križovec village (1 female, 12.06.2004, leg. F. Perović); Osijek city (3 males, 13.07.1901, leg. unknown); Pag Island, Povljana village (1 female, 30.05.1960, leg. unknown); Šenkovec village (1 male, 30.08.2009, leg. F. Perović); Southern Velebit Mountain, Struge-Buljma cabins, $1450 \mathrm{~m}$ a.s.1. (1 male, 25.06.1985, leg. B. Jalžić); Zagreb city, Bundek Lake (1 male, 24.05.1985, leg. unknown; 1 male, 12.07.2004, leg. F. Perović); Zrinska gora Mountain, Pecki Lake (1 male, 07.08.1916, leg. unknown).

\section{Coenagrion pulchellum (Vander Linden, 1825)}

The species is widely distributed in Europe but is scarcer in its southern part. It inhabits a wide range of lentic and slow flowing lotic habitats (lakes, ponds, marshes, slow-flowing ditches) (Askew, 2004; Dijkstra \& Lewington, 2006). In the collection, it is represented by a total of 12 individuals collected at seven localities in Croatia (CRO). The species is a protected (NAROdNe Novine, No. 80/13, 15/18) and nearly threatened taxon in Croatia (Belančić et al., 2008).

CRO: Goričan village, ponds by the state border (Croatia-Slovenia) (1 male, 15.03.2003, leg. F. Perović); Krapje Đol ornithological reserve (3 females, 16.05.1985, leg. unknown); Krka National Park, Roški slap waterfall (1 male, 21.05.1985, leg. unknown); Metković town, Neretva River (1 male, 1.05.1996, leg. unknown); Modro oko Lake (1 male, 28.06.1996, leg. M. Kučinić; 3 males, 08.04.1999, leg. F. Perović); Pag Island, Caska village (1 male, 31.05.2002, leg. F. Perović); Potok village, stream (1 male, 1 female, 07.06.2008, leg. F. Perović). 


\section{Coenagrion puella (Linnaeus, 1758)}

It is one of the most common species of the genus, being widespread in Europe to Asia. The species has wide ecological tolerance inhabiting wide range of lentic but also lotic habitats (AsKew, 2004; Dijkstra \& Lewington, 2006). In the collection, it is represented by a total of 74 individuals collected at 30 localities in Croatia (CRO).

CRO: Domašinec municipality, Murščak forest, Fučička pond (1 male, 26.05.2005, leg. F. Perović); Domašinec municipality, Murščak forest, pond (6 males, 3 females, 17.05.1999, leg. F. Perović; 2 males, 24.06.2005, leg. F. Perović); Domašinec municipality, Veliki Leš path, Murščak forest, canal (1 male, 23.05.2003, leg. F. Perović); Draganić municipality (1 male, 13.06.1964, leg. unknown; 1 male, 19.05.1975, leg. unknown; 3 males, 19.05.1978, leg. F. Perović); Goričan border crossing (Croatia-Slovenia), ponds (1 female, 25.06.2005, leg. F. Perović); Goričan village (2 males, 23.06.1999, leg. F. Perović); Goričan village, canal by the Mura River (2 males, 23.05.1999, leg. F. Perović); Goričan village, Trnava River mouth (1 male, 28.05.1999, leg. F. Perović); Goričan village, Trnava River, Crni bridge (1 male, 26.06.2005, leg. F. Perović); Goričan village, pond (1 male, 25.06.2005, leg. F. Perović); Gornji Mekinjar village, Murtino jezero Lake (3 males, 01.07.2004, leg. F. Perović); Gorski kotar region, stream (1 male, 19.06.1998, leg. unknown); Jurovčak village (1 male, 1 female, 30.05.1999, leg. F. Perović; 1 male, 26.05.2005, leg. F. Perović); Krapje Đol ornithological reserve (2 females, 16.05.1985, leg. unknown); Krbavsko polje, Močila village, springs (3 males, date unknown, leg. F. Perović); Križovec village, gravel pond (1 male, 1 female, 23.05.1999, leg. F. Perović; 1 male, 2 females, 17.03.2002, leg. F. Perović; 1 female, 29.05.2004, leg. F. Perović); Mikulići village (1 male, 08.1942, leg. unknown); Moslavačka gora Mountain, Mikleuška quarry (1 male, 13.06.1987, leg. S. Vujčić-Karlo); Motovun village, Butoniga Reservoir (2 males, 2 females, 16.06.1988, leg. D. Radović); Mura River, by the river ferry (1 female, 23.05.1999, leg. unknown); Repušnica village (3 males, 28.04.1983, leg. unknown; 5 males, 2 females, 21.05.1983, leg. unknown); Šenkovec village (1 male, 1 female, 05.10.2001, leg. F. Perović); Šenkovec village, Lateralac stream (1 female, 19.06.1999, leg. F. Perović); Stajničko polje, spring (3 males, 1 female, 28.06.2004, leg. F. Perović); Sveti Martin na Muri village (1 male, 1 female, 23.05.1999, leg. F. Perović); Turopolje wetland, Turska vrata locality (1 male, 27.05.1998, leg. M. Kučinić); Velika Gorica town (2 males, 07.1905, leg. unknown; 1 male, 09.05.1981, leg. unknown); Vugrovec village (2 males, 08.1942, leg. unknown); Zagreb city, Maksimir (1 female, 10.05.1981, leg. unknown).

\section{Coenagrion ornatum (Sélys, 1850)}

The species has a disjunct eastern Palearctic distribution, being very common in the south-east of the range in Europe and very local in the north and west of the range. It preferably inhabits sunny streams and flowing ditches with well-developed vegetation (AsKew, 2004; DijKstra \& Lewington, 2006). In the collection, it is represented by a total of 13 individuals collected at five localities in Croatia (CRO). The species is a nearly threatened taxon in Croatia (Belančić et al., 2008).

CRO: Domašinec municipality, Murščak forest, canal (1 male, 21.05.1998, leg. G. Gjerapić; 2 males, 1 female, 16.05.1999, leg. F. Perović; 1 male, 23.06.1999, leg. F. Perović); Domašinec municipality, Murščak forest, pond (1 male, 17.05.1999, leg. F. Perović); Goričan village, Trnava River mouth (1 male, 23.05.1999, leg. F. Perović; 1 male, 1 female, 28.05.1999, leg. F. Perović); Neretva River, Modro oko Lake (1 female, 08.04.1999, leg. F. Perović); Prekopa village (1 male, 2 females, 30.05.1999, leg. F. Perović). 


\section{Coenagrion scitulum (Rambur, 1842)}

The species is the commonest in the Mediterranean region of Europe and North Africa, being local in Central Europe, rare in Northern Europe and extending to the Middle East. It preferably inhabits sunny, lentic, sometimes slow flowing lotic habitats with well-developed aquatic vegetation (AsKew, 2004; DijKstra \& Lewington, 2006). In the collection, it is represented by a total of 12 individuals, 11of which were collected at eight localities in Croatia (CRO) and one individual at one locality in Serbia (SER).

CRO: Cres Island (1 male, 17.06.1997, leg. F. Perović); Dugopolje village, Sveti Anton church (1 male, 01.07.1996, leg. E. Kletečki); Istra, Vozilići village (2 males, 20.06.1975, leg. F. Perović; 1 male, 26.05.1977, leg. F. Perović; 1 male, 12.06.1977, leg. F. Perović); Krk Island, Njivice Reservoir (1 damaged specimen, 25.05.1924, leg. unknown); Lastovo Island, Lastovo polje, pond (2 males, 13.06.2001, leg. R. Ozimec); Neretva River (1 male, 01.05.1996, leg. F. Perović); Podolje village, pond (1 male, 13.06.1996, leg. G. Gjerapić), Vis Island, Podšpilje village, pond (1 damaged male, 14.06.1996, leg. F. Perović).

SER: Stara Pazova village (1 male, 22.07.1902, leg. unknown).

\section{Erythromma najas (Hansemann, 1823)}

The species is widely distributed in Northern and Central Europe but mainly absent from the Mediterranean region. Disjunct populations have been recorded in Asia from Turkestan to Japan. It preferably inhabits lentic habitats with well-developed floating vegetation. Sometimes it can be also found at slow flowing canals and dykes (AsKew, 2004; DiJKsTRA \& LEWINGTON, 2006). In the collection, it is represented by a total of five individuals collected at five localities in Croatia (CRO). The species is a protected (NARODNE NOvine, No. 80/13, 15/18) and nearly threatened taxon in Croatia (Belančić et al., 2008).

CRO: Križovec village, gravel pond (1 male, 29.05.2004, leg. F. Perović); Osijek city (1 male, 17.07.1901, leg. unknown); Osijek city, Halaševski canal (1 female, 10.05.1986, leg. M. Franković); Pag Island, Novalja town (1 male, 05.07.1960, leg. unknown); Unije Island (damaged specimen, 02.06.1964, leg. unknown).

\section{Erythromma viridulum (Charpentier, 1840)}

The species is mainly distributed in central and southern parts of Europe, North Africa and eastward as far as Turkestan. It preferably inhabits eutrophic lentic habitats with densely developed emergent and floating aquatic vegetation (AsKew, 2004; DiJKSTRA \& LEWINGTON, 2006). In the collection, it is represented by a total of three individuals collected at three localities in Croatia (CRO).

CRO: Goričan village (1 male, 09.08.1999, leg. F. Perović); Valpovo town, Gat village, 65 km from Drava River (1 female, 18.07.1989, leg. M. Franković); Zagreb city, Maksimir (1 male, 10.05.1981, leg. unknown).

\section{Erythromma lindenii (Sélys, 1840)}

The species is widespread from North Africa, across Southern Europe, expanding its area of distribution northwards. It inhabits larger, well-oxygenated lentic habitats (lakes, ponds, gravel pits) as well as slow flowing lotic habitats (rivers, canals) with well-developed aquatic vegetation (AsKew, 2004; DijKstra \& Lewington, 2006). In the collection, it is represented by a total of four individuals collected at four localities in Croatia (CRO). 
CRO: Blace village, Neretva River mouth (1 male, 29.04.1996, leg. F. Perović); Cetina River, Balečki bridge (1 female, 27.07.1985, leg. M. Franković); Krka National Park, Torak Lake (1 male, 03.07.1989, leg. M. Stipčević); Žumberak Mountain, Krašić village (damaged specimen, 16.07.1991, leg. M. Kučinić).

\section{Pyrrhosoma nymphula (Sulzer, 1776)}

The species is widely distributed in large parts of Northern and Central Europe, becoming more local towards the south. It inhabits a wide range of freshwater habitats, preferably lentic ones with well-developed vegetation (AsKEw, 2004; DiJKSTRA \& LEWINGTON, 2006). In the collection, it is represented by a total of 12 individuals collected at eight localities in Croatia (CRO).

CRO: Donji Mekinjar village, Stajnica spring (1 male, 03.07.2004, leg. N. Tvrtković); Goričan village, canal by the Mura River (1 male, 23.05.1999, leg. F. Perović); Jasenak village (2 males, 06.08.2005, leg. F. Perović); Jurovčak village (1 male, 30.05.1999, leg. F. Perović; 2 males, 25.06.2005, leg. F. Perović); Plitvice lakes (1 damaged specimen, 18.05.1985, leg. unknown); Potok village, stream (1 male, 07.06.2008, leg. F. Perović); Saborsko village (1 male, 1 female, 25.05.2004, leg. F. Perović); Stajničko polje, spring (1 male, 28.06.2004, leg. F. Perović).

\section{Ceriagrion tenellum (de Villers, 1789)}

The species is widespread in the western Mediterranean and locally common in the western part of Europe. It inhabits small streams and seepages, while in the northern part of the range it can be found mainly in bogs (AsKew, 2004; DiJKSTRA \& LeWINGTON, 2006). In the collection, it is represented by a total of three individuals collected at two localities in Croatia (CRO). The species is a strictly protected (NAROdnE Novine, No. 80/13, 15/18) and vulnerable taxon in Croatia (BELANčić et al., 2008).

CRO: Neretva River, Kuti Lake (2 males, 03.07.1997, leg. M. Kučinić); Neretva River, Modro oko Lake (1 male, 01.08.1997, leg. V. Ščetarić).

\section{Platycnemis pennipes (Pallas, 1771)}

The species has a wide European distribution, although it is absent from the Iberian Peninsula and mainly from Scandinavia. It inhabits a wide range of freshwater habitats, preferably well-vegetated slow flowing streams and rivers (AsKew, 2004; Dijkstra \& Lewington, 2006). In the collection, it is represented by a total of 120 individuals collected at 42 localities in Croatia (CRO).

CRO: Between Kožljak and Šunjevica villages, Boljunčica stream (1 male, 30.05.1984, leg. F. Perović); Domašinec municipality, Murščak forest (1 male, 1 female, 28.05.1999, leg. unknown); Domašinec municipality, Murščak forest, Mura River (1 female, 17.05.1999, leg. G. Gjerapić); Draganić municipality (1 male, 2 females, 13.06.1964, leg. unknown; 1 male, 13.05.1975, leg. F. Perović; 2 females, 19.05.1975, leg. F. Perović); Đurđevački pjesci sandstones (1 male, 1 female, 24.06.2003, leg. F. Perović); Glušci village, Neretva River (1 female, 30.06.1996, leg. M. Kučinić); Goričan village, Trnava River (1 male, 11.07.2000, leg. F. Perović); Goričan village, Trnava River, bridge (1 male, 26.06.2005, leg. F. Perović); Istra, Vozilići village (1 female, 29.06.1978, leg. F. Perović); Karlovac town (1 male, 1 female, 17.07.1902, leg. unknown; 3 females, 12.07.1904, leg. unknown); Krka National Park, Roški slap waterfall (1 male, 15.08.1999, leg. B. Jalžić); Kumrovec village (1 female, 24.06.1996, 
leg. F. Perović); Lonjsko polje wetland (1 female, 13.08.1982, leg. unknown); Lonjsko polje wetland, canal (1 male, 05.08.1982, leg. unknown); Matica River, Butina spring (1 female, 02.08.1996, leg. G. Gjerapić); Mislina village, Neretva River (1 female, 03.08.1996, leg. G. Gjerapić); Motovun forest (1 female, 03.08.1980, leg. Z. Bokulić); Motovun forest, water supply station near the meadows (1 male, 13.08.1980, leg. R.M.); Motovun village, five km downstream from the Butoniga Reservoir (1 female, date unknown, leg. D. Radović); Motovun village, Butoniga Reservoir (5 males, 6 females, 16.06.1988, leg. D. Radović); Motovun village, forest (1 male, 02.08.1980, leg. K.D.); Neretva River, Modro oko Lake (1 male, 6 females, 01.08.1997, leg. V. Šćetarić, G. Gjerapić; 1 male, 28.06.1996, leg. F. Perović; 1 male, 2 females, 04.08.1996, leg. M. Kučinić); 2 males, 2 females, 09.08.1997, leg. M. Kučinić), Obrovac town (1 male, 09.07.1931, leg. unknown); Ogulin town (1 male, 24.07.1914, leg. unknown); Opuzen town, Glavica quarry (2 males, 2 females, 29.06.1996, leg. F. Perović); Otok village, Čemec pond (1 male, 28.07.1907, leg. unknown); Paklenica Mountain (1 male, 20.06.1999, leg. F. Perović); Petrinja town (1 female, 25.05.1907, leg. unknown); Plitvice Lakes National Park, lakes (1 male, 21.09.1994, leg. F. Perović); Prevlaka village, near Rugvica (1 female, 20.05.1986, leg. F. Perović); Repušnica village (5 males, 4 females, 21.05.1983, leg. unknown); Selnica village (1 female, 21.09.1993, leg. F. Perović; 1 male, 1 female, 21.05.1999, leg. F. Perović; 1 male, 23.05.1999, leg. F. Perović; 1 female, 12.08.1999, leg. F. Perović; 1 male, 15.05.2004, leg. F. Perović); Turopolje wetland, Turska vrata locality (1 female, 27.05.1998, leg. M. Kučinić); Turopolje wetland, Vratovo locality (1 male, 01.07.2008, leg. D. Ćaleta); Zagreb city (1 female, 25.08.1899, leg. unknown); Zagreb city, Maksimir (1 female, 22.06.1952, leg. unknown); Zagreb city, Sava River (1 female, 08.06.1952, leg. unknown; 2 males, 1 female, 11.06.1992, leg. Z. Bokulić; 1 female, 21.06.1992, leg. unknown; 1 male, 26.06.1992, leg. unknown; 2 females, 27.06.1992, leg. unknown; 3 males, 1 female, 29.06.1992, Z. Bokulić; 1 male, 10.07.1992, leg. Z. Bokulić; 5 males, 3 females, 12.07.1992, leg. Z. Bokulić; 1 female, 19.07.1992, leg. unknown; 1 male, 21.07.1992, leg. Z. Bokulić; 1 male, 23.08.1992, leg. Z. Bokulić); Zagreb city, Savica (1 female, 02.05.1986, leg. D. Radović; 1 female, 04.05.1987, leg. D. Radović); Zapeć-Plemenitaš villages (1 female, 07.1909, leg. unknown; 1 male, 2 females, 07.08.1910, leg. unknown); Žumberak Mountain (1 female, 16.07.1991, leg. M. Kučinić).

\section{Suborder: ANISOPTERA Sélys, 1854}

\section{Family: AESHNIDAE}

\section{Aeshna mixta Latreille, 1805}

The species is widespread and common in large parts of Europe and reaches eastwards as far as Japan. It inhabits a wide range of lentic and slow-flowing lotic habitats with some riparian vegetation (DIJKSTRA \& LEWINGTON, 2006). In the collection, it is represented by a total of 24 individuals collected at 12 localities in Croatia (CRO).

CRO: Crna mlaka wetland (1 female, date and leg. unknown); Domašinec municipality, Murščak forest, Fučićka pond (1 male, date unknown, leg. F. Perović); Hvar Island, Poljica village (1 male, 22.08.1926, leg. unknown); Hvar Island, Starigrad village (1 female, 09.1910, leg. unknown); Medvednica Mountain, Sljeme summit (1 female, 12.08.1901, leg. unknown); Međimurje County, Šenkovec village (2 males, 05.10.2001, leg. F. Perović); Moslavačka gora Mountain, Mikleuška Lake (4 males, 2 females, 12.08.1982, leg. V. Jelenković); Osijek city (1 female, 17.08.1898, leg. unknown; 1 male, 24.08.1899, leg. unknown); Pag Island, Caska village (1 female, 01.10.1957, leg. unknown); Plitvice Lakes 
National Park (1 male, 18.08.1998, leg. unknown); Zagreb city (1 female, 06.08.1899, leg. unknown; 1 female, 17.08.1898, leg. unknown; 1 male, 12.09.1898; 1 female, 08.09.1899, leg. unknown; 1 male, 16.07.1901, leg. unknown; 1 female, date and leg. unknown); Zagreb city, Maksimir (1 male, date and leg. unknown).

\section{Aeshna affinis Vander Linden, 1820}

The species is most common in Southern and parts of Central Europe, while it is scarcer but recently increasing in the north of Europe (BоUdOT \& KALKMAN, 2015). Its area of distribution ranges eastwards to Mongolia. It preferably inhabits lentic habitats that dry out during summer and are overgrown with dense riparian vegetation (AsKEw, 2004; DiJKSTRA \& Lewington, 2006). In the collection, it is represented by a total of 15 individuals, 12of which were collected at ten localities in Croatia (CRO), one at one locality in Bosnia and Herzegovina (B\&H) and two individuals at one locality in Montenegro (MNE).

CRO: Kuterevo village, Švica Lake (1 male, 19.07.1912, leg. unknown); Lonjsko polje wetland, Provski potok stream (1 male, 25.07.1987, leg. unknown); Medvednica Mountain, Sljeme summit, Šumarev grob, Runolist (1 female, 20.07.1999, leg. unknown); Molve-Mače municipalities (1 male, 09.1928, leg. unknown); Neretva River, Modro oko Lake (1 male, 01.07.1996, leg. F. Perović); Osijek city (1 male, 11.05.1899, leg. unknown; 1 female, 06.07.1901, leg. unknown); Pag Island, Caska village (2 males, 06.07.2001, leg. F. Perović); Paklenica National Park (1 male, 20.07.1911, leg. unknown); Pelješac Peninsula, Monte Vipera, St. Ilija summit (1 female, 31.07.1995, leg. D. Holcer); Zagreb city, Maksimir (2 males, date and leg. unknown).

B\&H: Dinara Mountain, Poljanice village, 1000 m a.s.l. (1 female, 23.07.1987, leg. F. Perović).

MNE: Sekić village (Lovćenac village) (1 male, 1 female, 10.1933, leg. unknown).

\section{Aeshna isoceles (Müller, 1767)}

The species is widely distributed in the Mediterranean region and Central Europe, while it is very local in Northern Europe. It prefers lentic habitats (ditches, marshes, ponds, lakes) with well-developed vegetation (Askew, 2004; DijKstra \& Lewington, 2006). In the collection, it is represented by a total of six individuals collected at six localities in Croatia (CRO). The species is a protected (Narodne novine, No. 80/13, 15/18) and nearly threatened taxon in Croatia (BELANčić et al., 2008).

CRO: Crna mlaka wetland (1 male, 30.06.1907, leg. unknown); Domašinec municipality, Murščak forest, pond (1 male, 17.05.1999, leg. G. Gjerapić); Goričan village, pond by the state border (Croatia-Slovenia) (1 male, 16.05.2003, leg. G. Perović); Motovun village, Butoniga Reservoir (1 male, 16.06.1988, leg. D. Radović); Neretva River, lateral spring (1 male, 03.06.1993, leg. M. Stipčević); Sniježnica Konavoska mountain cliff, 800 m a.s.l. (1 female, 28.06.2000, leg. B. Jalžić).

\section{Aeshna grandis (Linnaeus, 1758)}

The species has a western Palearctic distribution. It is one of the most common species of the genus in Central and Eastern Europe. The eastern distribution spreads as far as Lake Baikal, while it is scarce and restricted towards the south of Europe. The species inhabits a wide range of lentic habitats with rich riparian and submerged vegetation, particularly in forested areas (AsKew, 2004; DiJKSTRA \& Lewington, 2006). In 
the collection, it is represented with one individual collected on the 09.07 .1922 by an unknown collector, at the locality Novi dvori of Zaprešić manor (CRO). The species is a strictly protected (NARODNE NOvine, No. 80/13, 15/18) and endangered taxon in Croatia (BELANČić et al., 2008).

\section{Aeshna cyanea (Müller, 1764)}

The species has a wide European distribution, from the Mediterranean region to the southern parts of Scandinavia. It inhabits a wide range of habitats, often in urban or heavily forested areas, but favours small shaded lentic habitats, often with no other substrate but leaf litter (AsKew, 2004; DijKstra \& Lewington, 2006). In the collection, it is represented by a total of 34 individuals, 32 of which were collected at 16 localities in Croatia (CRO), one at one locality in Bosnia and Herzegovina (B\&H) and one in Slovenia (SLO).

CRO: Bakar town (1 male, 12.09.1903, leg. unknown; 1 male, 12.11.1903, leg. unknown); Domašinec municipality, Murščak forest, canal (1 male, 06.10.2001, leg. F. Perović); Istra, Vozilići village, (1 male, 26.09.1977, leg. F. Perović); Jasenak village (3 males, 06.08.2005, leg. F. Perović); Lokve village (1 female, 07.1930, leg. unknown); Motovun village, road through the village (1 female, 05.08.1980, leg. Z. Bokulić); Northern Velebit National Park, Babrovača village (09.-13.10.1985, leg. F. Perović); Northern Velebit National Park, Zavižan summit (1 female, 06.07.1981, leg. Vukušić); Pelješac Peninsula, Monte Vipera, Sv. Ilija summit (1 male, 31.07.1995, leg. D. Holcer); Saborsko municipality, Plitvice Lakes National Park, Čorkova uvala forest (1 male, 18.09.2004, leg. F. Perović); Šenkovec, mouth of the stream into the retention (5 males, 1 female, 05.10.2001, leg. F. Perović); Temovec village (1 female, 07.1909, leg. I. von Igalffy; 1 female, 08.1925, leg. I. von Igalffy; 1 female, 08.1946, leg. I. von Igalffy; 1 female, date unknown, leg. I. von Igalffy); Velebit Mountain, Bubinica saddle, botanical reserve Sunđer (1 male, 19.08.1915, leg. unknown); Zagreb city (1 female, 17.09.1918, leg. unknown; 1 female, 12.08.1934, leg. unknown); Zagreb city, Maksimir (1 female, 12.08.1931, leg. unknown; 2 males, 3 females, date and leg. unknown); Zlobin village (1 female, 02.09.1915, leg. unknown).

B\&H: Dinara Mountain, Poparić pond, 1250 m a.s.1. (1 male, 24.07.1987, leg. S. Leiner).

SLO: Tržič village, Sv. Ana hill (1 male, 08.1932, leg. unknown).

\section{Anax imperator Leach, 1815}

The species is common and widespread in large parts of Western Eurasia and Africa. It inhabits large lentic habitats with well-developed vegetation (AsKEw, 2004; DiJKSTRA \& LEWINGTON, 2006). In the collection, it is represented by a total of 22 individuals, 21of which were collected at 18 localities in Croatia (CRO) and one individual at one locality in Bosnia and Herzegovina (B\&H).

CRO: Draganić municipality (1 female, 10.07.2004, leg. unknown); Istra, Grobnik village, (1 male, 23.05.1910, leg. unknown); Istra, Vozilići village (1 male, 07.1976, leg. F. Perović); Krk Island, Njivice Reservoir (1 male, date and leg. unknown); Lika County, Jablanac village (4 exuviae, 05.1932, leg. unknown); Lonjsko polje wetland, canal (1 male, 04.08.1982, leg. unknown); Međimurje County, Šenkovec municipality (1 male, 05.10.2001, leg. F. Perović); Motovun village, Butoniga Reservoir (1 male, 16.06.1988, leg. D. Radović); Osijek city (1 female, 17.07.1987, leg. unknown); Pag Island, Barbati village (1 female, 24.06.1956, leg. unknown); Pag Island, Zaglav village (1 female, 
26.06.1956, leg. unknown); Slavonija region, Trnovec village (1 male, date unknown, leg. Gj. Koča); Šenkovec municipality, mouth of the stream into the retention (1 male, 05.10.2001, leg. F. Perović); Unije Island (1 exuvia, 25.05.1924, leg. unknown); Vis Island (1 male, 07.-13.06.1910, leg. unknown); Vis Island, Podšpilje village, pond (1 female, 14.06.1996, leg. F. Perović); Zagreb city (1 male, 20.08.1899, leg. unknown); Zagreb city, Kajzerica, Sava River (1 female, 28.07.1907, leg. unknown).

B\&H: Vranjače village (1 female, 18.06.2002, leg. unknown).

\section{Anax parthenope (Sélys, 1839)}

The species is common in the Mediterranean region and scarcer towards the north of Europe. Its distribution ranges to Japan, China and Sahara. It inhabits large lentic habitats with well-developed vegetation (AsKew, 2004; Dijkstra \& Lewington, 2006). In the collection, it is represented by a total of three individuals collected at two localities in Croatia (CRO). The species is a protected (NARodne Novine, No. 80/13, 15/18) and nearly threatened taxon in Croatia (BELANČić et al., 2008).

CRO: Jablanac village (1 male, 04.-10.08.1908, leg. unknown); Pag Island, Velo Blato Lake (1 male, 04.07.1955, leg. unknown; 1 female, 05.05.2001, leg. G. Perović).

\section{Anax ephippiger (Burmeister, 1839)}

The species is typical for arid parts of Africa, the Middle East and south-west to Pakistan, but could also be sporadically found in Southern Europe. It inhabits shallow, warm pools and lakes, often with an intermittent character (AsKEw, 2004; DiJKsTRA \& LEWINGTON, 2006). In the collection, it is represented by a total of three individuals collected at two localities in Croatia (CRO). The species is strictly protected (NARODNE Novine, No. 80/13, 15/18) and vulnerable in Croatia (Belančıć et al., 2008).

CRO: Lonjsko polje wetland, Čigoč village (1 male, 1 female, 20.05.1989, leg. M. Franković); Osijek city, Petrijevci village (1 male, 19.07.1986, leg. M. Franković).

\section{Brachytron pratense (Müller, 1764)}

The species is widespread west of the Urals, generally localised and rare in the south of Europe. It inhabits lotic or lentic habitats with well-developed riparian and aquatic vegetation (favours e.g. reedy canals, marshes, oxbows) (Askew, 2004; Dijkstra \& LEWINGTON, 2006). In the collection, it is represented by a total of five individuals collected at five localities in Croatia (CRO).

CRO: Goričan village, pond by the state border (Croatia-Slovenia) (1 male, 16.05.2003, leg. F. Perović); Medvednica Mountain, Sljeme summit (1 male, 25.05.1908, leg. unknown); Modro oko Lake (1 male, 08.04.1999, leg. F. Perović); Turopolje wetland, Greda village (1 male, 03.05.1995, leg. F. Perović); Velika Jasenovača village (1 male, 20.05.1904, leg. unknown).

\section{Family: GOMPHIDAE}

\section{Gomphus vulgatissimus (Linnaeus, 1758)}

It is the most common species of the genus in most of Northern and Central Europe, with its distribution area ranging eastward to the Urals. The species typically inhabits 
slow-flowing streams and rivers with a sandy bottom but can also be found in large lakes (AsKew, 2004; DijKstra \& Lewington, 2006). In the collection, it is represented by a total of 13 individuals collected at ten localities in Croatia (CRO).

CRO: Klanjec town (1 female, 06.06.1931, leg. unknown); Krka National Park, Roški slap waterfall (1 female, 21.05.1985, leg. unknown); Odra River (1 male, 04.05.1980, leg. F. Perović); Petrinja town (2 males, 1 female, 24.05.1907, leg. unknown); Pešćenica village, Odra River (1 female, 08.03.1977, leg. unknown); Temovec village (1 male, 05.1927, leg. I. von Igalffy; 1 female, 07.1938, leg. I. von Igalffy); Turopolje wetland, Turopoljski lug, Vratovo locality (1 female, 25.05.1997, leg. G. Gjerapić); Turopolje wetland, Greda village (1 female, 09.07.1986, leg. F. Perović); Turopolje wetland, Pešćenica village (1 male, 28.05.1995, leg. F. Perović); Zagreb city (1 female, 10.05.1993, leg. unknown).

\section{Ophiogomphus cecilia (Geoffroy in Fourcroy, 1785)}

The species is widespread over most of Eastern Europe up to Germany, while in the southern regions it can be found locally. It inhabits lotic habitats from small highland rivers to large lowland rivers, most typically the lower reaches of large rivers with sandy bottoms (AsKew, 2004; DijKstra \& LeWINGTON, 2006). In the collection, it is represented by a total of eight individuals collected at five localities in Croatia (CRO). The species is a strictly protected (NARODNE NOvine, No. 80/13, 15/18) and endangered taxon in Croatia (BELANČić et al., 2008).

CRO: Kalnik Mountain (1 male, 04.08.1917, leg. unknown; 1 female, 06.08.1917, leg. unknown); Klek Mountain (2 males, 25.07.1914, leg. unknown); Ravna gora village (1 female, 03.06.1918, leg. unknown); Zagreb city (1 male, 01.07.1898, leg. unknown); Zagreb city, Trnje (2 males, 17.07.1908, leg. unknown).

\section{Onychogomphus forcipatus (Linnaeus, 1758)}

The species is widespread across Europe, very common in the south but local in the north. The area of distribution ranges to the Urals. It typically inhabits unshaded streams and rivers with rocky bottoms but can also be found in clear lakes (AsKEw, 2004; DiJKsTRA \& Lewington, 2006). In the collection, it is represented by a total of eight individuals collected at six localities in Croatia (CRO).

CRO: Odra River (1 female, 25.04.2010, leg. F. Perović); Plitvice Lakes National Park (1 male, 18.08.1998, leg. unknown); Plitvice Lakes National Park, Poljanak village (1 female, 03.08.2005, leg. F. Perović); Sošice village (1 male, 22.06.1917, leg. unknown); Zagreb city (1 female, 09.06.1898, leg. unknown; 1 male, 1 female, 08.1933, leg. unknown); Zrinska gora Mountain, Majdan village (1 male, 11.07.1987, leg. F. Perović).

\section{Lindenia tetraphylla (Vander Linden, 1825)}

The species has a disjunct distribution from central Asia, through the Middle East to the Mediterranean basin. In Europe, there are only a few permanent populations, restricted to the lakes and larger slow flowing rivers in the Mediterranean region. Its typical habitats are large lakes and slow flowing rivers with well-developed reedbeds (AsKEw, 2004; Boudot \& Kalkman, 2015, Dijkstra \& Lewington, 2006). In the collection, it is represented by a total of seven individuals collected at four localities in Croatia (CRO). The species is a strictly protected (NARODNE NOvine, No. 80/13, 15/18) and endangered taxon in Croatia (Belančić et al., 2008). 
CRO: Metković town, Bobaj hill, Kula Norinska (1 male, 29.06.2000, leg. R. Ozimec); Neretva River, Kuti Lake/oxbow (1 female, 03.07.1996, leg. M. Kučinić); Pag Island, Povljana village (2 males, 06.1962, leg. unknown); Pag Island, Velo blato Lake (3 males, 28.06.1962, leg. unknown).

\section{Family: CORDULEGASTRIDAE}

\section{Cordulegaster bidentata Sélys, 1843}

The species is endemic to Europe, having a patchy distribution and being more common in the Balkan Peninsula than in other parts of Europe. It preferably inhabits small shallow streams in hilly and mountainous regions (AsKEw, 2004; DiJKSTRA \& Lewington, 2006). In the collection, it is represented by a total of nine individuals, seven of which were collected at six localities in Croatia (CRO) and two at one locality in Slovenia (SLO).

CRO: Biokovo Mountain, Gornje Igrane village (1 exuvia, 18.06.1990, leg. F. Perović); Istra, Vozilići village (1 female, 31.05.1975, leg. F. Perović); Kalnik Mountain (1 male, 1 female, 04.08.1917, leg. unknown); Medvednica Mountain, Sljeme summit (1 male, 09.07.1911, leg. unknown); Zagreb city (1 female, 23.06.1917, leg. unknown); Zrinska gora Mountain, Majdan village (1 male, 12.07.1987, leg. F. Perović).

SLO: Radeče town (2 females, 07.1929, leg. unknown).

\section{Family: CORDULIIDAE}

\section{Cordulia aenea (Linnaeus, 1758)}

The species is common and widespread in Northern Eurasia, while it is more localised in the southern part of Europe. It inhabits lentic habitats, such as large ponds, small lakes, bogs, oxbows, gravel pits, fishponds, canals (AsKew, 2004; DijKsTRA \& LEWINGTON, 2006). In the collection, it is represented by a total of 83 individuals collected at seven localities in Croatia (CRO).

CRO: Domašinec municipality, Murščak forest, canal (1 female, 19.05.2001, leg. unknown); Domašinec municipality, Murščak forest, pond (1 male, 17.05.1999, leg. G. Gjerapić); Dubrovnik city (16 males, 20 females, 41 exuviae, 07.-17.05.1994, leg. unknown); Grubišno polje town (1 female, 22.05.1910, leg. unknown); Osijek city (1 female, 15.04.1997, leg. unknown); Velika Gorica town (1 female, 22.04.1981, leg. unknown); Zagreb city, Borongaj (1 male, 03.05.1901, leg. unknown).

\section{Somatochlora metallica (Vander Linden, 1825)}

The species is widespread and common from Central Europe eastward to Central Siberia. In Southern Europe, it is restricted to mountain lakes. It inhabits various lentic and slow flowing lotic habitats (e.g. ponds, oxbows, moorland lakes, sluggish rivers) (Askew, 2004; Dijkstra \& Lewington, 2006). In the collection, it is represented by one male individual collected on 01.08 .1980 by F. Perović at the locality Durmitor Mountain, Žabljak village, Crno jezero Lake in Montenegro (MNE). In Croatia, the species is considered to be regionally extinct (BELANČić et al., 2008). 


\section{Somatochlora meridionalis Nielsen, 1935}

The species is nearly endemic to Europe, where it is most abundant in its south-eastern part, especially the Balkan Peninsula. It inhabits lotic habitats, most commonly heavily shaded lowland streams and rivers (Askew, 2004; Boudot \& Kalkman, 2015, Dijkstra \& Lewington, 2006). In the collection, it is represented by a total of ten individuals collected at eight localities in Croatia (CRO).

CRO: Čakovec town (1 female, 14.07.2004, leg. unknown); Hvar Island (1female, 23.07.1914, leg. unknown); Mljet Island, Malo jezero Lake (1 female, 12.08.1982, leg. unknown); Motovun village, Butoniga Reservoir (1 female, 16.06.1988, leg. D. Radović); Pag Island, Kolansko blato wetland (1 female, 11.06.1958, leg. unknown); Pešćenica village (1 female, 07.08.1978, leg. D. Perović); Southern Velebit Mountain, below Štirovac summit, Ćorina prosina meadow (1 female, 18.07.1985, leg. F. Perović); Zagreb city, Maksimir (1 male, 08.1942, leg. unknown; 2 males, date and leg. unknown).

\section{Somatochlora flavomaculata (Vander Linden, 1825)}

The species is generally rare, having a mainly Central and East European distribution, with its range extending to Mongolia. It can be found at lentic habitats (such as small ponds, marshes, boggy meadows, dykes, ditches) at low altitudes, often also in cultivated areas (AsKew, 2004; DijKstra \& Lewington, 2006). In the collection, it is represented by a total of two individuals collected at two localities in Croatia (CRO). The species is a protected (NARODNE NOvine, No. 80/13, 15/18) and nearly threatened taxon in Croatia (BELANČić et al., 2008).

CRO: Krbavsko polje, Močila village, springs (1 male, 09.07.2005, leg. F. Perović); Krka River (1 male, 26.06.1996, leg. F. Perović).

\section{Epitheca bimaculata (Charpentier, 1825)}

It is a North Eurasian species, occurring eastward as far as Japan, and has a very patchy distribution in Europe. The species could be found at small and rather deep lakes with well-developed vegetation, oxbows, gravel pits, fishponds (AsKEw, 2004; DiJKsTRA \& Lewington, 2006). In the collection, it is represented by a total of three individuals collected at two localities in Croatia (CRO). The species is a strictly protected (NARodNE Novine, No. 80/13, 15/18) and endangered taxon in Croatia (Belančić et al., 2008).

CRO: Osijek city (1 male, 1 female, 22.04.1997, leg. unknown); Turopolje wetland, Pešćenica village (1 male, 28.05.1995, leg. F. Perović).

\section{Family: LIBELLULIDAE}

\section{Libellula quadrimaculata Linnaeus, 1758}

The species has Holarctic distribution, being widespread through North America and Eurasia. Preferably inhabits lentic habitats with well-developed vegetation (AsKEw, 2004; DijKstra \& Lewington, 2006). In the collection, it is represented by a total of three individuals collected at three localities in Croatia (CRO).

CRO: Gorski kotar region, Ličko Polje (1 male, 26.06.1910, leg. unknown); Northern Velebit National Park, Žive Vodice pond, 1270 m a.s.l. (1 male, 04.08.1984, leg. F. Perović); Zagreb city, Jarun Lake (1 male, 11.06.1989, leg. M. Franković). 


\section{Libellula depressa Linnaeus, 1758}

The species is among the most common and most widespread European odonates, extending to central Asia. It inhabits a wide range of lentic habitats, preferably small, shallow, sunny and bare ones (AsKew, 2004; DijKstra \& LeWINGTON, 2006). In the collection, it is represented by a total of 55 individuals collected at 35 localities in Croatia (CRO).

CRO: Dijaneš village (2 males, date and leg. unknown); Domašinec municipality, Murščak forest, canal (1 male, 26.06.2005, leg. unknown); Draganić municipality (2 males, 03.06.1964, A. Magerle); Dubrovnik city (1 female, 30.05.1985, leg. Halapir); Goričan village (1 male, 23.06.1999, leg. F. Perović); Gorski kotar region, Ravna Gora municipality, Šije village, (1 female, 05.06.1982, leg. B. Jalžić); Gospić town (1 male, 10.08.1915, leg. unknown); Istra, meadows (1 female, 20.06.1997, leg. F. Perović); Istra, Vozilići village (1 female, 09.06.1976, leg. F. Perović; 1 female, 11.07.1976, leg. F. Perović); Jurovčak village (1 female, 26.05.2005, leg. F. Perović); Krbavsko polje, Močila village (1 male, 30.07.2004, leg. F. Perović); Krk Island, Njivice Reservoir (3 males, 1 female, 25.05.1924, leg. unknown); Kupa River, quarry (1 male, 09.06.2006, leg. F. Perović); Lokrum Island (across the Dubrovnik city) (2 males, 3 females, 30.05.1985, leg. Halapir); Marijanci village (1 female, 29.06.1906, leg. unknown); Motovun village, Butoniga Reservoir (1 male, 16.06.1988, leg. D. Radović); Northern Velebit National Park, Žive Vodice pond, 1270 m a.s.l. (1 female, 13.06.1983, leg. F. Perović); Osijek city (1 female, 05.05.1997, leg. unknown; 2 females, 30.04.98, leg. unknown); Pag Island, Lun village (1 male, 11.10.1955, leg. unknown); Pag Island, Stara Povljana bay (1 female, 10.05.1986, leg. B. Jalžić); Pag Island, Velo blato Lake (1 female, 28.06.1962, leg. unknown); Pag Island, Vidalići village, Barbati (1 male, 06.1956, leg. unknown); Pag Island, Zaglav village (1 male, 26.06.1956, leg. unknown); Pešćenica village (1 female, 08.05.1995, leg. F. Perović); Petrinja town (1 female, 25.05.1907, leg. unknown); Repušnica village, canal (1 female, 21.05.1983, leg. unknown); Temovec village (1 female, 07.1925, leg. I. von Igalffy; 1 female, 08.1944, leg. I. von Igalffy; 1 female, 05.1956 , leg. I. von Igalffy); Trnovec village (1 male, 08.1955, leg. unknown; 1 male, 07.1956, leg. I. von Igalffy); Velika Gorica town (1 female, 07.05.1981, leg. unknown); Vrpolje village (1 female, 15.07.1912, leg. unknown); Zagreb city (1 female, 20.04.1933, leg. unknown; 3 females, 05.1933, leg. unknown); Zagreb city, Bundek Lake (1 male, 24.05.1985, leg. unknown); Zagreb city, Maksimir (3 females, date and leg. unknown); Zrinska gora Mountain (1 male, 11.07.1987, leg. S. Vujčić-Karlo); Žumberak Mountain, Sveta Gera summit (1 male, 20.06.1917, leg. unknown).

\section{Libellula fulva Müller, 1764}

The species is widespread in Europe, but as it has specific habitat preferences, its distribution is local. The female requires open water surface (without floating vegetation) for oviposition, therefore it preferably inhabits slow-flowing streams and rivers, canals, reedy-lakes, fishponds and oxbows (AsKew, 2004; DijKstra \& Lewington, 2006). In the collection, it is represented by a total of 14 individuals collected at nine localities in Croatia (CRO).

CRO: Domašinec municipality, Murščak forest, canal (5 females, 16.05.1999, leg. F. Perović); Krka National Park, Visovac Lake (2 males, 25.04.1989, leg. D. Radović); Matica River, Butina spring (1 female, 01.05.1996, leg. F. Perović); Neretva River, Desne Lake (1 male, 07.04.1999, leg. F. Perović); Neretva River, Kuti Lake/oxbow (1 male, 03.07.1996, leg. M. Kučinić); Neretva River, Modro oko Lake (1 male, 28.06.1996, leg. F. Perović); 
Pešćenica village (1 female, 22.05.1994, leg. F. Perović); Selce village, Odra River (1 female, 06.05.1990, leg. F. Perović); Turopolje wetland (1 female, date and leg. unknown).

\section{Orthetrum cancellatum (Linnaeus, 1758)}

The species is common and widespread in Europe (except northern Scandinavia), North Africa, extending to Kashmir and Mongolia. It is typically found in lowlands, where it inhabits larger lentic or slow-flowing lotic habitats with unvegetated margins (AsKew, 2004; DijKstra \& Lewington, 2006). In the collection, it is represented by a total of 33 individuals, 32 of which were collected at 24 localities in Croatia (CRO) and one at one locality in Austria (AUT).

CRO: Baćinska jezera lakes, Lokvenica Lake (1 male, 02.08.1997, leg. F. Perović); Bijeli vir village, Neretva River (1 female, 02.08.1996, leg. F. Perović; 1 female, 08.08.1996, leg. F. Perović); Biograd na moru town (1 male, 06.07.1927, leg. T. Šoljan); Blace village, Neretva River (1 male, 1 female, 26.07.1997, leg. F. Perović, G. Gjerapić); Borovci village, Neretva River (1 female, 08.08.1997, leg. M. Kučinić); Glušci village, Neretva River (1 female, 03.08.1996, leg. G. Gjerapić); Hvar Island, Starigrad village (1 female, 08.1996, leg. unknown); Jablanac village (1 male, 05.07.1908, leg. unknown); Jurovčak village (1 exuvia, 30.05.1999, leg. F. Perović); Matica River, Butina spring (1 male, 2 females, 02.08.1996, leg. G. Gjerapić); Motovun village, Butoniga Reservoir (1 female, 16.06.1988, leg. D. Radović); Mura River (1 female, 23.06.1999, leg. G. Perović); Neretva River, Modro oko Lake (1 female, 28.06.1996, leg. M. Kučinić); Opuzen town, Glavica quarry (2 females, 29.06.1996, leg. F. Perović); Pag Island, bridge (1 damaged female, 22.07.1988, leg. M. Stipčević); Pag Island, Caska village (1 male, 27.06.1960, leg. unknown); Pag Island, Kolan village (1 male, 27.06.1916, leg. unknown); Pag Island, Kolansko blato wetland (2 males, 01.07.1955, leg. unknown); Pag Island, Malo blato Lake (1 female, 03.07.1955, leg. unknown); Pag Island, Novalja town, Vidasi (2 males, 1 female, 28.07.1996, leg. F. Perović, G. Gjerapić); Pag Island, Povljana village (1 male, 03.07.1956, leg. unknown); Tenja village (1 male, 15.07.1901, leg. unknown); Vrana village (1 female, 20.07.1927, leg. T. Šoljan); Zagreb city (1 male, 03.06.1899, leg. unknown).

AUT: Wien city, Prater park (1 female, 06.07.1905, leg. unknown).

\section{Orthetrum albistylum (Sélys, 1848)}

This predominantly eastern Palearctic species has a patchy distribution from Europe to China and Japan. It inhabits open ponds and lakes, often with very scarce aquatic vegetation (AsKew, 2004; DijKstra \& LEWINGTon, 2006). In the collection, it is represented by a total of 16 individuals, 14 of whichwere collected at nine localities in Croatia (CRO) and two at two localities in Serbia (SER).

CRO: Between villages Jastrebarsko and Draganići, fishpond (1 male, 05.06.1989, leg. V. Bartovski); Crna mlaka wetland (1 male, 30.05.1907, leg. unknown); Draganić municipality (1 male, 13.05.1963, leg. unknown; 1 female, 03.06.1964, leg. unknown; 1 female, 13.06.1964, leg. unknown; 1 male, 10.07.2004, leg. F. Perović); Lonja village, Lonja River (1 male, 1984, leg. V. Jelenković); Osijek city, Bajeri lakes (1 female, 14.06.2002, leg. F. Perović); Selnica village (1 female, 12.06.2004, leg. unknown); Selnica village, fishpond (1 male, 23.05.1999, leg. F. Perović; 1 male, 1 female, 23.06.1999, leg. F. Perović); Temovec village (1 male, 07.1925, leg. I. von Igalffy); Zagreb city (1 female, 01.07.1899, leg. unknown). 
SER: Kupinovo village (1 female, 11.08.1913, leg. unknown); Subotište village (1 male, 25.07.1902, leg. unknown).

\section{Orthetrum coerulescens (Fabricius, 1798)}

The species is common in Mediterranean region while its distribution in Central and Northern Europe is rather local. It can also be found in North Africa and Asia (as far as to northern India). Typically inhabits lotic habitats, such as streams and ditches, and in the northern part of its range occurs in brooks in boggy areas (AsKEw, 2004; DiJKSTRA \& Lewington, 2006). In the collection, it is represented by a total of 56 individuals collected at 25 localities in Croatia (CRO). The species is a protected (NAROdNE Novine, No. 80/13, 15/18) and data deficient taxon in Croatia (Belančić et al., 2008).

CRO: Bijeli vir village, Neretva River (1 male, 02.08.1996, leg. F. Perović); Blace village (1 female, 27.07.1997, leg. G. Gjerapić; 1 female, 28.07.1997, leg. V. Ščetarić; 2 females, 29.07.1997, leg. V. Ščetarić); Blace village, Neretva River mouth (1 female, 26.07.1997, leg. G. Gjerapić; 2 females, 29.07.1997, leg. V. Dumbović; 1 female, 01.08.1997, leg. V. Ščetarić; 2 females, 10.09.1997, leg. F. Perović); Blace village, Obli vir (1 female, 08.08.1996, leg. F. Perović), Borovci village (1 male, 08.08.1997, leg. unknown); Borovci village, Neretva River (1 male, 26.07.1997, leg. G. Gjerapić; 5 males, 2 females, 31.07.1997, leg. V. Dumbović, V. Ščetarić); Borovci village, Vratar castle, Neretva River (1 female, 03.08.1996, leg. F. Perović); Dretulja River spring (2 females, 01.07.2004, leg. N. Tvrtković; 2 females, 28.07.2004, leg. F. Perović; 1 male, 30.07.2004, leg. unknown); Glušci village, Neretva River (1 female, 29.06.1996, leg. M. Kučinić); Goričan village, Trnava River, bridge (1 male, 26.06.2005, leg. F. Perović); Komin village, north from the school, Neretva River (1 male, 2 females, 09.08.1996, leg. F. Perović); Končanica town (1 male, 01.08.1918, leg. unknown); Kostanjevac village (1 female, 13.07.1918, leg. unknown); Krka River (1 male, 25.06.1996, leg. B. Jalžić); Matica River, Butina spring (2 females, 02.08.1996, leg. G. Gjerapić); Mislina village, Neretva River (2 males, 03.08.1996, leg. G. Gjerapić); Motovun village, meadow (1 female, 02.08.1980, leg. unknown); Neretva River (1 female, 08.08.1997, leg. M. Kučinić); Neretva River, Modro oko Lake (1 male, 28.06.1996, leg. M. Kučinić; 1 female, 01.07.1996, leg. F. Perović; 1 male, 09.08.1997, leg. M. Kučinić); Opuzen town, Glavica quarry (1 male, 1 female, 29.06.1996, leg. F. Perović); Opuzen town, Mala Neretva River (6 males, 30.07.1997, leg. V. Dumbović, V. Ščetarić); Pag Island, Kolansko blato wetland (1 male, 17.06.1958, leg. unknown); Pag Island, Lun village, Vidasi, pond (1 female, 03.05.2001, leg. F. Perović); Plešce village (1 male, 17.07.03, leg. unknown); Plitvice lakes (1 male, 21.09.2004, leg. F. Perović).

\section{Orthetrum brunneum (Fonscolombe, 1837)}

In Europe, the species is most common in the Mediterranean area, is local in Central Europe and absent from its northern part. It also occurs in North Africa, the Middle East and Asia eastward to Mongolia. This lowland species preferably inhabits small lotic habitats, such as streams, ditches, seepages (AsKew, 2004; DiJKsTrA \& Lewington, 2006). In the collection, it is represented by a total of 22 individuals, 21 of which were collected at nine localities in Croatia (CRO) and one at one locality in Serbia (SER).

CRO: Biokovo Mountain, Kotišina village (1 male, 27.07.1995, leg. F. Perović); Draga village (1 male, date and leg. unknown); Istra, Vozilići village (1 male, 24.07.1975, leg. F. Perović; 1 male, 11.07.1976, leg. unknown); Osijek city (1 male, 17.07.1901, leg. unknown); Virovitica town (1 female, date unknown, leg. Polašek); Vis Island (1 male, 
07.-13.06.1910, leg. unknown); Zagreb city (1 male, 05.06.1899, leg. unknown; 1 male, 1 female, 11.06.1898, leg. unknown; 1 male, 17.06.1899, leg. unknown; 2 males, 30.06.1898, leg. unknown; 1 male, 01.07.1899, leg. unknown; 1 female, 30.05.1908, leg. unknown; 1 male, 1 female, 23.06.1908, leg. unknown; 2 females; date unknown; leg. S. V. Weingartner); Zagreb city, Maksimir (1 male, date and leg. unknown); Zagreb city, Sava River (1 female, 23.08.1992, leg. unknown).

SER: Subotica city (1 male, 25.07.1902, leg. unknown).

\section{Leucorrhinia pectoralis (Charpentier, 1825)}

This Euro-Siberian species is generally uncommon in Europe having local and small populations. Southwards in Europe it reaches as far as the Balkans, Turkey, Northern Italy and French Pyrenees. It prefers marshy borders to bogs, forest lakes, fenlands, oxbows, small canals with dense growth of Typha and Carex (Askew, 2004; Dijkstra \& Lewington, 2006). In the collection, it is represented with one female collected on 30.04.1998 by an unknown collector, at the locality Osijek city in Croatia (CRO). The species is a strictly protected (NARODNE NOvine, No. 80/13, 15/18) and endangered taxon in Croatia (BELANČIć et al., 2008).

\section{Sympetrum danae (Sulzer, 1776)}

The distribution of this Circumboreal species ranges to Japan and across North America. In Europe it is mainly distributed in its northern part, while towards the south it becomes more local and restricted to higher altitudes. It preferably inhabits acidic lentic habitats, such as bogs, moorland and heathy lakes (DIJKSTRA \& LEWINGTON, 2006). The collection contains one male collected on April $8^{\text {th }} 1899$, but as the name of the locality was missing, we were not able to determine where the specimen/species originates from.

\section{Sympetrum pedemontanum (Müller in Allioni, 1766)}

The species is generally distributed in Central and Southern Europe, but is rare in the Balkan Peninsula. It becomes more common towards the east to Japan. Habitat preferences are still insufficiently studied but it is considered to be associated with hilly areas where it inhabits seepages, slow flowing lotic habitats with well-developed vegetation, and sunny shallow pools (AsKew, 2004; DijKstra \& Lewington, 2006). In the collection, it is represented by a total of two individuals collected at two localities in Croatia (CRO). The species is strictly protected (NAROdne Novine, No. 80/13, 15/18) and critically endangered taxon in Croatia (Belančıć et al., 2008) (Fig. 2).

CRO: Goričan village (1 male, 09.08.1999, leg. G. Perović); Goričan village, Črni most bridge (1 male, 09.08.1999, leg. G. Perović).

\section{Sympetrum sanguineum (Müller, 1764)}

The species is common and widespread throughout the Europe and eastward to Siberia, where it inhabits most freshwater habitats with well-developed marshy vegetation (Askew, 2004; Dijkstra \& Lewington, 2006). In the collection, it is represented by a total of 111 individuals, 101 of which were collected at 44 localities in Croatia $(\mathrm{CRO})$, seven individuals at five localities in Bosnia and Herzegovina $(\mathrm{B} \& \mathrm{H})$ and three individuals at three localities in Serbia (SER). 
CRO: Babina greda village (1 male, 1 female, 17.07.1912, leg. unknown); Between the villages Molve and Mače (1 male, 09.1928, leg. unknown); Borovci village, Vratar castle, Neretva River (2 females, 03.08.1996, leg. F. Perović); Bregana town (1 female, 16.05.1901, leg. unknown); Bregi village (1 male, 17.07.1900, leg. unknown); Dekanovec Village, Murščak forest, canal (3 males, 1 female, 26.06.2005, leg. F. Perović); Dekanovec Village, Murščak forest, ponds (1 male, 19.09.1999, leg. G. Perović); Dinara Mountain, Crvene grede, $1000 \mathrm{~m}$ a.s.l. (1 female, 24.07.1985, leg. M. Franković); Dinara Mountain, Crvene grede, $800 \mathrm{~m}$ a.s.l. (1 female, 27.07.1985, leg. M. Franković); Dobranje village, Neretva River (1 female, 06.08.1996, leg. F. Perović); Draganić municipality (1 female, 03.06.1964, leg. unknown); Dubravica village, Neretva River (3 males, 03.08.1996, leg. G. Gjerapić); Dubrovnik-Neretva County, Goršćaki village (1 male, 09.08.1999, leg. F. Perović); Glušci village, Neretva River (2 males, 1 female, 03.8.1996, leg. G. Gjerapić); Goričan village (1 male, 05.09.2003, leg. F. Perović); Istra, Butoniga Reservoir (1 male, 10.09.1988, leg. M. Stipčević); Karlovac town (1 male, 29.08.1909, leg. unknown); Konjščina village (1 male, 21.07.1997, leg. F. Perović); Krk Island (1 male, 09.08.1922, leg. unknown); Krbavsko polje, Močila village (1 male, 30.07.2004, leg. F. Perović); Krka National Park, Roški slap waterfall (1 female, 15.08.1999, leg. B. Jalžić); Lonjsko polje wetland (1 male, 2 females, 09.08.1982, leg. V. Jelenković; 1 female, 13.08.1982, leg. unknown; 1 female, 01.08.1992, leg. unknown); Lonjsko polje wetland, canal (1 male, 02.08.1982, leg. unknown); Matica River, Butina spring (1 male, 02.08.1996, leg. G. Gjerapić); Mislina village, Neretva River (1 male, 03.08.1996, leg. G. Gjerapić); Motovun village, pond in the forest (1 male, 06.08.1880, leg. K. E.); Mura River (1 female, 11.08.99, leg. unknown); Neretva River, Modro oko Lake (1 male, 04.08.1996, leg. M. Kučinić; 1 male, 01.08.1997, leg. F. Perović); Opuzen town, Glavica quarry (1 male, 1 female, 29.06.1996, leg. F. Perović); Osijek city (1 male, 1 female, 19.07.1901, leg. unknown; 2 males, 05.08.1901, leg. unknown; 2 males, 02.08.1997, leg. unknown; 1 male, 03.08.1998, leg. unknown; 1 female, 22.08.1998, leg. unknown); Pag Island, Caska village (1 female, 23.10.1956, leg. unknown); Paklenica National Park, Štirovac summit (4 females, 07.08.1954, leg. unknown); Repušnica village, canal by the highway (1 male, 1 female, 13.08.1982, leg. unknown); Repušnica village, forest by the highway (1 male, 13.08.1983, leg. unknown); Slavonija region, near Našice city, Lilin dvor (1 male, 11.08.1915, leg. unknown; 1 male, 11.08.1959, leg. unknown); Sunja village (1 female, 31.08.1911, leg. unknown); Šenkovec village (1 male, 05.10.2001, leg. F. Perović); Štigrova village (2 males, 5 females, 07.08.1954, leg. unknown); Turopolje wetland, Greda village (1 female, 09.07.1986, leg. F. Perović); Velebit Mountain, Lomska duliba, 1200 m a.s.l. (1 female, 09.08.1994, leg. unknown); Zagreb city (1 male, 12.08.1898, leg. unknown; 1 male, 06.09.1899, leg. unknown; 2 males, 23.06.1908, leg. unknown; 1 male, 11.09.1950, leg. unknown; 4 females, 11.09.1951, leg. unknown; 1 male, 11.09.1957, leg. unknown; 4 males, 5 females, date and leg. unknown); Zagreb city, Maksimir (1 male, 1 female, 22.06.1952, leg. unknown; 1 male, 21.09.1954, leg. unknown; 3 males, date and leg. unknown); Zapeć-Plemenitaš villages (2 males, 07.08.1910, leg. unknown); Zrinska gora Mountain, Majdan village (1 female, 11.07.1987, leg. S. Vujčić-Karlo).

B\&H: Bosanski Petrovac municipality, Bara village (1 male, 12.10.1999, leg. unknown); Dinara Mountain, Poljanice village (1 female, 23.07.1987, leg. F. Perović; 1 female, 13.07.1988, leg. B. Jalžić); Dinara Mountain, Poljanice village, $1200 \mathrm{~m}$ a.s.l. (1 female, 23.07.1987, leg. S. Leiner); Dinara Mountain, Poljanice-Troglav, $1200 \mathrm{~m}$ a.s.1. (1 female, 23.07.1987, leg. F. Perović); Dinara Mountain, Troglav, Poparić pond, $1250 \mathrm{~m}$ a.s.1. (1 male, 1 female, 24.07.1987, leg. S. Leiner). 
SER: Beočin town (1 male, 20.07.1917, leg. unknown); Vitojevci village (1 male, 10.08.1913, leg. unknown); Zeleni breg hill (1 male, 05.08.1905, leg. unknown).

\section{Sympetrum depressiusculum (Sélys, 1841)}

This is the most localized species of the genus, having a patchy distribution in Central and Southern Europe, extending to eastern Asia (Japan). As it has poor dispersing abilities, it is often found close to the breeding habitats, such as rice paddies, fishponds, intermittent lakes and cooling-water basins (AsKew, 2004; DiJKsTRA \& LEWINGTON, 2006). In the collection, it is represented by one female collected at the locality Zagreb city in Croatia (CRO) by an unknown collector and at an unknown date. The species is a strictly protected (NARODNE NOvine, No. 80/13, 15/18) and critically endangered taxon in Croatia (Belančić et al., 2008).

\section{Sympetrum flaveolum (Linnaeus, 1758)}

The species is common in most of Southern, Central and Eastern Europe, extending to Siberia, Kamchatka and Japan. Patchy distributed in Balkan and Iberian Peninsulas. Preferably inhabits shallow and well-vegetated habitats, such as swampy depressions and seasonally flooded meadows (Askew, 2004; Dijkstra \& Lewington, 2006). In the collection, it is represented by a total of 11 individuals, two of which were collected at two localities in Croatia (CRO), eight individuals at four localities in Bosnia and Herzegovina $(\mathrm{B} \& \mathrm{H})$ and one individual at one locality in Switzerland $(\mathrm{CH})$. The species is a strictly protected (NARODNE NOvine, No. 80/13, 15/18) and vulnerable taxon in Croatia (BELANČić et al., 2008).

CRO: Krbavsko polje, Gornja Močila village, springs (1 female, 09.07.2005, leg. F. Perović); Vis Island, Podšpilje village, sandstones (1 male, 04.09.1997, leg. F. Perović).

B\&H: Dinara Mountain (Vučevica area), Dubac village, Goleš summit, $1550 \mathrm{~m}$ a.s.l. (2 females, 26.07.1985, leg. M. Franković); Dinara Mountain, Poljanice village, $1200 \mathrm{~m}$ a.s.l. (1 female, 23.07.1987, leg. S. Leiner; 2 females, 13.07.1988, leg. M. Kučinić); Dinara Mountain, Poparić pond (2 females, 23.07.1987, leg. F. Perović); Livanjsko polje, Kazanci-Provo villages (1 female, 25.07.1987, leg. F. Perović).

CH: Mönchalptal bei Davos (1 female, 29.08.1953, leg. unknown).

\section{Sympetrum fonscolombii (Sélys, 1840)}

The species is widely distributed in warm parts of Africa and Asia, common in the Mediterranean but rare in the northern part of Europe. It inhabits warm, open, bare and shallow lentic habitats, such as quarry lakes, sand pits, newly created ponds and coastal lagoons (Askew, 2004; Dijkstra \& Lewington, 2006). In the collection, it is represented by a total of 22 individuals collected at 14 localities in Croatia (CRO). The species is a protected (NARODNE NOVINE, No. 80/13, 15/18) and nearly threatened taxon in Croatia (BELANČić et al., 2008).

CRO: Draganić municipality (2 males, 13.06.1964, leg. unknown); Goričan village, Mura River oxbow, Hušićeva pond (5 males, 26.10.2009, leg. F. Perović); Korčula Island, Lumbarda village (1 male, 1 female, 14.08.2003, leg. F. Perović); Krk Island, Jezero Reservoir (1 female, 17.06.1998, leg. unknown); Međimurje County, Šenkovec village (1 male, 05.10.2001, leg. F. Perović); Neretva River, Modro oko Lake (1 female, 28.06.1996, leg. M. Kučinić; 1 female, 09.08.1997, leg. M. Kučinić); Palagruža Island (1 female, 
15.06.1996, leg. F. Perović); Svetac Island (1 female, 09.06.1997, leg. F. Perović); Unije Island (1 male, 02.06.1964, leg. unknown); Vela Palagruža Island (1 male, 1 female, 12.07.1996, leg. F. Perović); Vis Island, Komiža town (Mala Palagruža) (1 male, 12.06.1996, leg. unknown); Vis Island, Podšpilje village, pond (1 female, 13.06.1996, leg. G. Gjerapić); Vis Island, Podšpilje village, sandstones (1 male, 04.09.1997, leg. F. Perović); Zagreb city (female, date and leg. unknown).

\section{Sympetrum striolatum (Charpentier, 1840)}

The species has a wide European distribution, extending to Japan. It inhabits a wide range of habitats, favouring warm lentic ones. The species is very often a pioneer of newly created ponds (AsKew, 2004; Dijkstra \& Lewington, 2006). In the collection, it is represented by a total of 83 individuals, 82 of which were collected at 33 localities in Croatia (CRO) and one individual at one locality in Bosnia and Herzegovina (B\&H).

CRO: Baška voda village (1 female, 08.09.1978, leg. F. Perović); Blace village, Neretva River (1 female, 10.09.1997, leg. F. Perović); Blace village, Neretva River mouth (1 female, 26.07.1997, leg. G. Gjerapić; 2 females, 19.10.1997, leg. F. Perović); Dobranje village, Neretva River (1 female, 06.08.1996, leg. F. Perović); Domašinec municipality, canal (1 male, 1 female, 06.10.2001, leg. unknown); Domašinec municipality, Murščak forest, Fučička pond (1 male, 15.10.2005, leg. G. Perović); Domašinec municipality, Murščak forest, canal (1 female, 24.10.2009, leg. F. Perović); Domašinec municipality, Murščak forest, Veliki Leš path (4 females, 13.11.2009, leg. F. Perović); Goričan village, Hušićeva pond (1 female, 26.10.2009, leg. F. Perović); Goričan village, Trnovec stream (1 male, 10.1956, leg. unknown); Istra, Vozilići village (1 female, 26.07.1974, leg. unknown; 1 male, 20.06.1975, leg. unknown; 1 female, 24.07.1975, leg. unknown; 1 female, 18.08.1975, leg. F. Perović; 1 female, 20.08.1975, leg. unknown; 1 male, 1 female, 03.10.1975, leg. F. Perović; 1 female, 26.09.1977, leg. unknown); Ivanja Reka-Hrušćica villages (1 male, 23.11.1986, leg. M. Franković); Jagnjedovac village (1 female, 18.07.1900, leg. unknown); Krapina town (1 male, 25.08.1914, leg. unknown); Krk Island, Njivice Reservoir (1 female, 25.05.1924, leg. unknown); Krk Island, Ponikve Reservoir (1 male, 1 female, 19.08.1926, leg. unknown); Lekenik village, Kalje forest (1 female, 17.06.1994, leg. F. Perović); Pag Island, Caska village (1 female, 06.10.1955, leg. unknown; 1 female, 09.06.1958, leg. unknown; 1 male, 1 female, 12.06.1958, leg. unknown; 1 male, 31.05.2002, leg. F. Perović); Pag Island, Kolansko blato wetland (1 male, 5 females, 11.06.1958, leg. unknown); Paklenica Mountain, Male Močile village, $150 \mathrm{~m}$ a.s.1. (1 female, 21.06.1985, leg. F. Perović); Plitvice Lakes National Park (1 female, 18.08.98, leg. unknown); Plješevica Mountain (1 male, 15.08.1918, leg. unknown); Pustodol village (1 female, 01.10.1986, leg. unknown); Rijeka city (1 female, 23.09.1997, coll. unknown); Svetac Island (1 female, 09.06.1996, leg. G. Gjerapić); Trenovac village (Nova Ljeskovica nowadays) (1 female, 08.1985, leg. I. von Igalffy); Trnovec village, Drava River oxbow (1 male, 1 female, 02.11.2001, leg. F. Perović); Vis Island, Podšpilje village, pond (3 males, 4 females, 13.06.1996, leg. F. Perović, G. Gjerapić; 1 female, 14.06.1996, leg. F. Perović); Vis Island, pond (1 female, 02.01.1988, leg. Z. Ružanović); Zagreb city (9 males, 9 females, date and leg. unknown), Zagreb city, Maksimir (1 male, 21.09.1954, leg. unknown; 1 female, date and leg. unknown); Zagreb city, Prevlaka village, Odra river (1 damaged specimen, 23.09.1985, leg. M. Franković); Zagreb city, Sava River (1 female, 11.06.1992, leg. Z. Bokulić).

B\&H: Miljacka River (1 male, 26.10.2001, leg. F. Perović). 


\section{Sympetrum vulgatum (Linnaeus, 1758)}

The species is common in Central, Northern and Eastern Europe, extending to Siberia and China. The distribution is scarcer and more disjunct in Southern Europe. It inhabits a wide range of lentic habitats with well-developed vegetation (AsKew, 2004; Dijkstra \& LeWington, 2006). In the collection, it is represented by a total of 22 individuals collected at ten localities in Croatia (CRO). The species is a protected (NARodnE NOvine, No. 80/13, 15/18) and nearly threatened taxon in Croatia (BELANČić et al., 2008).

CRO: Dekanovec village, Murščak forest, Fučička pond (1 male, 03.08.1998, leg. G. Gjerapić; 1 male, 03.10.2003, leg. F. Perović; 1 male, 15.10.2005, leg. G. Perović); Dekanovec village, Murščak forest, pond (2 males, 19.09.1999, leg. F. Perović); Goričan village (1 male, 09.08.1999, leg. F. Perović); Goričan village, canal by the Trnava River, Črni most bridge (1 female, 20.04.1999, leg. G. Perović; 2 males, 1 female, 20.09.1999, leg. unknown); Karlovac town (1 female, 29.08.1909, leg. unknown); Krbavsko polje, Močila village (1 female, 30.07.2004, leg. F. Perović); Pag Island, Caska village (1 female, 16.06.1958, leg. unknown); Šenkovec village, fishpond (1 female, 22.06.2008, leg. F. Perović); Trnovec village, Drava River (1 female, 13.10.2001, leg. unknown; 1 male, 02.11.2001, leg. F. Perović); Zagreb city (1 male, 14.10.1909, leg. unknown; 3 males, 2 females, date and leg. unknown).

\section{Sympetrum meridionale (Sélys, 1841)}

The species is common in the south of Europe but also found in Central Europe. It also occurs in North Africa, and eastwards from the Middle East to Mongolia and South-Eastern Siberia. It prefers shallow, well-vegetated lentic habitats, such as sunny pools, muddy oxbows and seasonal swamps (Askew, 2004; DijKstra \& Lewington, 2006). In the collection, it is represented by a total of 44 individuals, 37 of which were collected at 32 localities in Croatia (CRO), five individuals at four localities in Serbia (SER) and two individuals at one locality in Montenegro (MNE). The species is a protected (NARodne Novine, No. 80/13, 15/18) and nearly threatened taxon in Croatia (BELANČić et al., 2008).

CRO: Blace village (1 female, 29.07.1997, leg. V. Ščetarić); Blace village, Neretva River mouth (1 female, 26.07.1997, leg. G. Gjerapić; 1 female, 29.07.1997, leg. V. Dumbović; 1 female, 01.08.1997, leg. V. Dumbović; 1 female, 10.09.1997, leg. V. Strpić; 1 female, 10.09.1997, leg. F. Perović; 1 male, 19.10.1997, leg. F. Perović; 1 female, 04.08.2003, leg. F. Perović); Dobranje village, Neretva River (1 female, 06.08.1996, leg. F. Perović); Krk Island (1 male, 2 females, 08.08.1922, leg. unknown); Krk Island, Ponikve Reservoir (1 male, 19.08.1926, leg. unknown); Krndija Mountain (1 damaged individual, 27.07.1916, leg. unknown); Kutjevo town (1 male, 28.07.1916, leg. unknown); Mala Neretva River (1 female, 30.07.1997, leg. V. Ščetarić); Modro oko Lake (1 female, 04.08.1996, leg. M. Kučinić); Motovun village, road by the meadows (1 male, 13.08.1980, leg. R. M.); Osijek city (1 female, 11.07.1901, leg. unknown; 1 male, 05.08.1901, leg. unknown; 1 female, 08.08.97, leg. unknown); Pag Island, Kolansko blato wetland (1 male, 11.06.1958, leg. unknown); Pag Island, ponds (1 female, 07.07.2001, leg. unknown); Pag Island, village Caska (1 male, 09.06.1958, leg. unknown; 1 male, 12.06.1958, leg. unknown; 2 females, 06.07.2001, leg. F. Perović; 1 female, 31.08.2001, leg. F. Perović); Štrigova village (2 females, 07.08.1954, leg. unknown); Vinkovci town (1 male, 1 female, 29.08.1912, leg. unknown); Zagreb city (3 males, 1 female, date and leg. unknown); Zagreb city, Remete (1 female, 11.09.1957, leg. unknown). 
SER: Beočin town (1 male, 20.07.1917, leg. unknown); Čortanovci village (1 male, 27.08.1908, leg. unknown); Fruška gora (2 males, 08.07.1904, leg. unknown); Kupinovo village (1 male, 19.07.1902, leg. unknown).

MNE: Kotor town (2 females, 07.1931, leg. unknown).

\section{Crocothemis erythraea (Brullé, 1832)}

The species is common and widely distributed in Africa, Western Asia and most of Europe, expanding its area of distribution northwards. It inhabits all lentic habitats (AsKew, 2004; DijKsTRA \& LeWINGTON, 2006). In the collection, it is represented by a total of 45 individuals, 44 of which were collected at 28 localities in Croatia (CRO) and one at one locality in Italy (ITA).

CRO: Baćinska jezera lakes, Lokvenica Lake (1 female, 02.08.1997, leg. F. Perović); Blace village (1 female, 28.07.1997, leg. V. Ščetarić); Blace village, Neretva River mouth (1 female, 26.07.1997, leg. G. Gjerapić); Crna mlaka wetland (1 male, 30.06.1907, leg. unknown); Draganić village (1 male, 10.07.2004, leg. F. Perović); Draganić village, fishpond (2 males, 10.07.2004, leg. F. Perović); Goričan village (1 female, 09.08.1999, leg. F. Perović); Hvar Island, Starigrad village (1 male, 08.1910, leg. unknown); Križovec village (2 males, 12.08.1999, leg. F. Perović, G. Perović); Krk Island, Njivice Reservoir (2 males, 18.08.1926, leg. unknown); Krka River (1 male, 26.06.1996, leg. F. Perović); Lastovo Island, pond (1 male, 13.06.2001, leg. R. Ozimec); Mislina village, Neretva River (2 males, 03.08.1996, leg. G. Gjerapić); Motovun village, Butoniga Reservoir (1 male, 16.06.1988, leg. D. Radović); Neretva River, Kuti Lake (1 male, 1 female, 03.07.1996, leg. M. Kučinić); Osijek city (1 female, 13.05.1901, leg. unknown; 1 male, 22.08.1998, leg. unknown); Pag Island, Caska village (1 male, 27.06.1960, leg. unknown; 1 female, 28.06.1960, leg. unknown); Pag Island, Kolan village (1 male, 27.06.1956, leg. unknown); Pag Island, Novalja town (1 male, 14.07.1908, leg. unknown); Pag Island, Novalja town, Vidasi (4 males, 1 female, 28.07.1996, leg. G. Gjerapić); Pag Island, ponds (1 male, 07.07.2001, leg. F. Perović); Rijeka city, Trsat hill (1 male, 07.07.1904, leg. unknown; 1 male, 07.07.1907, leg. unknown); Savski Đol wetland (near Sisak town) (1 male, 29.07.1916, leg. unknown); Unije Island (1 female, 28.06.1963, leg. unknown; 1 male, 1 female, 02.09.1964, leg. unknown); Vis Island, Podšpilje village, pond (1 male, 13.06.1996, leg. F. Perović); Vis Island, Podšpilje village, sandstones (1 male, 04.09.1997, leg. S. Leiner); Vrana village (1 male, 20.07.1927, leg. unknown); Zagreb city (1 female, 06.08.1899, leg. G. Gjerapić; 1 male, 20.08.1899, leg. unknown; 1 male, date and leg. unknown).

ITA: Trieste city (1 male, 07.07.1904, leg. unknown).

\section{Selysiothemis nigra (Vander Linden, 1825)}

The species mainly occurs in central Asia and the Middle East, while its European distribution is mainly restricted to isolated populations in the Mediterranean region. It can also be found in Africa, being localized to oases in Sahara. It inhabits shallow lentic habitats (Askew, 2004; Dijkstra \& Lewington, 2006). In the collection, it is represented by a total of three individuals collected at three localities in Croatia (CRO). The species is a strictly protected (NARODNE NOvine, No. 80/13, 15/18) and endangered taxon in Croatia (BElančić et al., 2008).

CRO: Mala Neretva River (1 female, 30.07.1997, leg. V. Ščetarić); Mislina village, Neretva River (1 female, 03.08.1996, leg.G. Gjerapić); Opuzen town, Glavica quarry (1 female, 29.06.1996, leg. F. Perović). 


\section{CONCLUSION}

Almost all Odonata species occurring in Croatia (84\%) are represented in the collection of the Croatian Natural History Museum. As most of the previously published data on Croatian Odonata originate from the Croatian mainland, this collection is valuable since there is a number of specimens and species from Croatian islands. Moreover, the collection contains a number of endangered and protected Odonata species from both Croatia and surrounding countries, such as Lindenia tetraphylla, Somatochlora metallica, Leucorrhinia pectoralis, Selysiothemis nigra, Sympetrum pedemontanum, S. depressiusculum etc. This collection is of essential importance in further Odonata research and conservation practices in this part of Europe, as comparison of historical and newly observed data allows us to detect possible changes in species distribution, as well as changes in their natural habitats.

\section{ACKNOWLEDGEMENTS}

The authors would like to thank the reviewers for their useful comments and suggestions that markedly improved this manuscript.

Received March 12, 2018

\section{REFERENCES}

Askew, R. R., 2004: The dragonflies of Europe, Second Edition, Harley Books, Essex, 308 pp.

Belančić, A., Bogdanović, T., Franković, M., Ljuština, M., Minoković, N. \& Vitas, B., 2008: Crvena knjiga vretenaca Hrvatske. Ministarstvo kulture, Državni zavod za zaštitu prirode Republike Hrvatske, Zagreb, 132 pp. (Red data book of dragonflies of Croatia. Ministry of Culture, State Institute for Nature protection, Zagreb, Croatia, 132 pp). (In Croatian).

BeLle, J., 1994: Some dragonfly records from the Lesser Sunda island on Bali and Lombok, Indonesia, with an ethno-odonatological note. Notulae odonatologicae 4, 60-62.

Bogdanović, T., Merdić, E. \& Mikuska, J., 2008: Data to the dragonfly fauna of the lower Neretva River. Entomologia Croatica 12(2), 51-65.

Boudot, J.-P. \& Kalkman, V. J. (eds.), 2015: Atlas of the European dragonflies and damselflies. KNNV publishers, the Netherlands, 381 pp.

Carrara, F., 1846: La Dalmazia descritta. Fratteli Battala tipografi editori, Zara.

D'Aguilar, J., Dommanget, J. L. \& Prechac, R., 1986: A field guide to the dragonflies of Britain, Europe and North Africa. William Collins Sons and Company Ltd, London, 336 pp.

Dijkstra, K.-D. B. \& Lewington, R., 2006: Field Guide to the Dragonflies of Britain and Europe. British Wildlife Publishing, Gillingham, 320 pp.

Grimaldi, D. \& Engel, M. S., 2005: Evolution of the Insects. Cambridge University press, New York, 755 pp.

Jović, M. \& Minajlova, B., 2009: Catalogue of the Odonata collection in the Macedonian Museum of Natural History. Acta Entomologica Serbica 14(2), 133-146.

Kalkman, V. J., Clausnitzer, V., Dijkstra, K-D. B., Orr, G. A., Paulson, R. D. \& Tol, J.V., 2008: Global diversity of dragonflies (Odonata) in freshwater. Hydrobiologia 595, 351-363.

Kalkman, V. J., Boudot, J.-P., Bernard, R., Conze, K.-J., de Knijf, G., Dyatlova, E., Ferreira, S., Jović, M., Ott, J., Riservato, E. \& Sahlén, G., 2010: European Red List of Dragonflies. Publications Office of the European Union, Luxembourg, 28 pp.

Kotarac, M., 1997: Atlas of the Dragonflies (Odonata) of Slovenia with the Red Data List: a Project of the Slovene Dragonfly Society. Atlas faunae et florae Sloveniae 1. Center za kartografijo favne in flore, Miklavž na Dravskem polju, 205 pp.

Kotarac, M., Šalamun, A., Vilenica, M., 2016: Projekt integracije u EU Natura 2000 - Terensko istraživanje i laboratorijska analiza novoprikupljenih inventarizacijskih podataka za taksonomske grupe: Actinopterygii i Cephalaspidomorphi, Amphibia i Reptilia, Aves, Chiroptera, Decapoda, Lepidopte- 
ra, Odonata, Plecoptera, Trichoptera / EU Natura 2000 Integration Project (IBRD No. 8021-HR) - Field research and laboratory processing for collecting new inventory data for taxonomic groups: Actinopterygii and Cephalaspidomorphi, Amphibia and Reptilia, Aves, Chiroptera, Decapoda, Lepidoptera, Odonata, Plecoptera, Trichoptera - Završno izvješće za taksonomsku skupinu Odonata / Final report for the taxonomic group Odonata, $39 \mathrm{pp}$.

Kulijer, D., De Knijf, G. \& Franković, M., 2013: Review of the Odonata of Bosnia and Herzegovina. Odonatologica 42(2), 109-123.

Moore, N. W, 1997: Status survey and conservation action plan. Dragonflies. IUCN/SSC Odonata Specialist Group. IUCN, Gland and Cambridge, 28 pp.

Narodne Novine, 2018: Zakon o zaštiti prirode, pročišćeni tekst NN 80/13, 15/18. Pravilnik o strogo zaštićenim vrstama. Narodne Novine 80/13, 15/18. Available from https://www.zakon.hr/z/403/Zakon-o-zaštiti-prirode (accessed on 11/03/2018). [Official Gazette, 2018: Ordinance on Strictly Protected Wild Taxa. Official Gazette 80/13, 15/18: Available from https://www.zakon.hr/z/403/Zakon-o-zaštiti-prirode (accessed on 11/03/2018).]

Perović, G. \& Perović, F., 2006: Preliminarni rezultati istraživanja vretenaca (Odonata) na području Međimurja, Hrvatska. (Preliminary results of research into dragonflies (Odonata) in Međimurje, Croatia). Entomologia Croatica 10(1-2), 87-103. (In Croatian).

Schorr, M. \& PAulson, D., 2018: World Odonata List. https://www.pugetsound.edu/academics/academic-resources/slater-museum/biodiversity-resources/dragonflies/world-odonata-list2/ (Accessed on 15/05/2018).

Simaika, J. P. \& Samways, M. J, 2008: Valuing dragonflies as service providers. In: Cordoba-Aguilar, A. (ed.), Dragonflies: Model Organisms for Ecological and Evolutionary Research. Oxford University Press, Oxford, UK. p. 109-123.

Suhling, F., SÀhlen, G., Martens, A., Marais, E. \& Schütte, C., 2006: Dragonfly assemblages in arid tropical environments: a case study from western Namibia. Biodiversity and Conservation 15, 311332.

ŠAšić, M., NAhirnić, A. \& TARmann, G. M., 2016: Zygaenidae (Lepidoptera) in the Lepidoptera collections of the Croatian Natural History Museum. Natura Croatica 25(2), 233-248.

Štih, A., Koren, T., Bobinec, A., Matejčıć, M. \& Franković, M., 2015: The River Zrmanja - Another Hotspot of Dragonfly Diversity in the Dinaric Karst, Croatia. Entomologia Croatica 19(1-2), 43-57.

VilenicA, M. \& DijKstra, K.-D. B, 2014: The dragonfly (Insecta, Odonata) fauna of the Banovina region, Croatia. Natura Croatica 23(1), 45-66.

Vilenica, M., Mičetić Stanković, V. \& Franković, M., 2011: Dragonfly fauna (Insecta, Odonata) in the Turopolje region (Croatia). Natura Croatica 20(1), 141-15.

Vilenica, M., Alegro, A., Koletić, N. \& Mihaljević, Z., 2016: New evidence of Lindenia tetraphylla (Vander Linden, 1825) (Odonata, Gomphidae) reproduction at the north-western border of its distribution. Natura Croatica 25(2), 287-294.

Vinko, D. \& VILENICA, M., 2013: BOOM 2013: Third Balkan odonatological Meeting: Tretje mednarodno srečanje odonatologov Balkana Hrvaška, 1.-7. Avgust 2013: Rezultati. Erjavecia 28, 10-19.

Vinko, D., 2017: BOOM 2017, 7th Balkan odonatological meeting, 7. mednarodno srečanje odonatologov Balkana, Slovenija, 4.-11. Avgust 2017. Erjavecia 32, 29-40. 